\title{
ANALYTIC OPERATOR LIPSCHITZ FUNCTIONS IN THE DISK AND A TRACE FORMULA FOR FUNCTIONS OF CONTRACTIONS
}

\author{
M.M. MALAMUD, H. NEIDHARDT AND V.V. PELLER
}

To the memory of M.S. Agranovich, a remarkable mathematician and a remarkable personality

\begin{abstract}
In this paper we prove that for an arbitrary pair $\left\{T_{1}, T_{0}\right\}$ of contractions on Hilbert space with trace class difference, there exists a function $\boldsymbol{\xi}$ in $L^{1}(\mathbb{T})$ (called a spectral shift function for the pair $\left.\left\{T_{1}, T_{0}\right\}\right)$ such that the trace formula $\operatorname{trace}\left(f\left(T_{1}\right)-\right.$ $\left.f\left(T_{0}\right)\right)=\int_{\mathbb{T}} f^{\prime}(\zeta) \boldsymbol{\xi}(\zeta) d \zeta$ holds for an arbitrary operator Lipschitz function $f$ analytic in the unit disk.
\end{abstract}

\section{Introduction}

The notion of spectral shift function was introduced by physicist I.M. Lifshits in [L]. Later M.G. Krein elaborated the notion of spectral shift function in [Kr1] a most general situation; he showed that for self-adjoint operators $A_{0}$ and $A_{1}$ with trace class difference, there exists a unique real function $\boldsymbol{\xi}=\boldsymbol{\xi}_{A_{1}, A_{0}}$ in $L^{1}(\mathbb{R})$ (it is called the spectral shift function for the pair $\left.\left\{A_{1}, A_{0}\right\}\right)$ such that the following trace formula holds:

$$
\operatorname{trace}\left(f\left(A_{1}\right)-f\left(A_{0}\right)\right)=\int_{\mathbb{R}} f^{\prime}(t) \boldsymbol{\xi}_{A_{1}, A_{0}}(t) d t
$$

for sufficiently nice functions $f$.

To prove the existence of the spectral shift function, he introduced the concept of perturbation determinant $\Delta_{A_{1} / A_{0}}$ and proved the inversion formula

$$
\boldsymbol{\xi}_{A, A_{0}}(t)=\frac{1}{\pi} \lim _{y \downarrow 0} \operatorname{Im}\left(\log \left(\Delta_{A_{1} / A_{0}}(t+\mathrm{i} y)\right)\right) \quad \text { for a.e. } \quad t \in \mathbb{R},
$$

where $\Delta_{A_{1} / A_{0}}(\zeta) \stackrel{\text { def }}{=} \operatorname{det}\left(I+\left(A-A_{0}\right)\left(A_{0}-\zeta I\right)^{-1}\right)$ (see $[\mathrm{Kr} 1]$ and $\left.[\mathrm{Ya}]\right)$.

Later Krein extended in $[\mathrm{Kr} 3]$ formula $(1.1)$ to the class $W_{1}(\mathbb{R})$ of functions whose derivative is the Fourier transform of a complex Borel measure.

Krein also observed in [Kr1] that the right-hand side of (1.1) makes sense for arbitrary Lipschitz functions $f$ and posed the problem to describe the class of functions, for which formula (1.1) holds for all pairs of self-adjoint operators with difference of trace class $\boldsymbol{S}_{1}$.

It turned out that trace formula (1.1) cannot be generalized to the class of all Lipschitz functions. Indeed, it was shown in $[\mathrm{F}]$ that there exist a Lipschitz function $f$ on $\mathbb{R}$ and self-adjoint operators $A_{1}$ and $A_{0}$ such that $A_{1}-A_{0} \in \boldsymbol{S}_{1}$, but $f\left(A_{1}\right)-f\left(A_{0}\right) \notin \boldsymbol{S}_{1}$. In [Pe1] and [Pe3] it was proved that (1.1) holds for functions $f$ in the (homogeneous) Besov space $B_{\infty, 1}^{1}(\mathbb{R})$ and does not hold unless $f$ locally belongs to the Besov space $B_{1,1}^{1}$

Krein's problem was completely resolved recently in [Pe5]. It was shown in [Pe5] that trace formula (1.1) holds for arbitrary pairs $\left\{A_{1}, A_{0}\right\}$ of not necessarily bounded 
self-adjoint operators with trace class difference if and only if $f$ is an operator Lipschitz function, i.e., the inequality

$$
\|f(A)-f(B)\| \leq \text { const }\|A-B\|
$$

holds for arbitrary self-adjoint operators $A$ and $B$.

In [Kr2] Krein introduced the notion of spectral shift function for pairs of unitary operators with trace class difference. Namely, for a pair of unitary operators $\left\{U_{1}, U_{0}\right\}$ with trace class difference, he proved that there exists a real function $\boldsymbol{\xi}$ in $L^{1}(\mathbb{T})$, unique modulo an additive constant, (called a spectral shift function for $\left\{U_{1}, U_{0}\right\}$ ) such that the trace formula

$$
\operatorname{trace}\left(f\left(U_{1}\right)-f\left(U_{0}\right)\right)=\int_{\mathbb{T}} f^{\prime}(\zeta) \boldsymbol{\xi}(\zeta) d \zeta
$$

holds for functions for $f$ whose derivative $f^{\prime}$ has absolutely convergent Fourier series.

In the recent paper [AP2] an analog of the result of [Pe5] was obtained: the class of functions $f$, for which formula (1.2) holds for arbitrary pairs $\left\{U_{1}, U_{0}\right\}$ of unitary operators with $U_{1}-U_{0} \in \boldsymbol{S}_{1}$ coincides with the class $\mathrm{OL}_{\mathbb{T}}$ of operator Lipschitz functions on $\mathbb{T}$.

We are going to extend in this paper trace formulae (1.1) and (1.2) to the case of pairs of maximal dissipative operators (m-dissipative) and pairs of contractions.

The Sz.-Nagy-Foiaş functional calculus (see [SNF]) associates with each function $f$ in the disk-algebra $\mathrm{C}_{\mathrm{A}}$ (i.e., the space of functions analytic in the disk $\mathbb{D}$ and continuous in its closure) the operator $f(T)$. This functional calculus is linear and multiplicative and the von Neumann inequality $\|f(T)\| \leq \max \{|f(\zeta)|: \zeta \in \mathbb{C},|\zeta| \leq 1\}$ holds for $f \in \mathrm{C}_{\mathrm{A}}$.

When we proceed to the case of contractions, it is natural to divide the problem in two problems: (i) the existence of a spectral shift function and a trace formula for resolvents; (ii) a description of the largest class of functions for which the trace formula holds.

First generalizations of formula (1.1) to the case of pairs $\left\{A_{1}, A_{0}\right\}$ with an m-accumulative (dissipative) operator $A_{1}$ were obtained by Rybkin [R1] and Krein [Kr4]. For instance, Krein treated the case when $A_{0}=A_{0}^{*}, A_{1}=A_{0}-\mathrm{i} V, V \geq 0$ and $V \in \boldsymbol{S}_{1}$ and proved in [Kr4] an analog of formula (1.1) with right-hand side $\int_{\mathbb{R}} f^{\prime}(t) d \nu(t)$ for a complex Borel measure $\nu$ and for functions $f$ of class $W_{1}^{+}$, i.e., functions $f$ whose derivative is the Fourier transform of a complex measure supported in $[0, \infty)$. The most complete result on the existence of a spectral shift function for pairs $\left\{L_{1}, L_{0}\right\}$ of maccumulative resolvent comparable operators satisfying $\rho\left(L_{0}\right) \cap \mathbb{C}_{+} \neq \varnothing$ was obtained in [MN2]. Under these assumptions, formula (1.1) was proved for resolvents. In the case of additive $\boldsymbol{S}_{1}$ perturbations trace formula (1.1) was proved for the class $W_{1}^{+}$(see [MN2]).

The purpose of this paper is to prove that for a pair $\left\{T_{1}, T_{0}\right\}$ of contractions satisfying $T_{0}-T_{1} \in \boldsymbol{S}_{1}$, there exists a function $\boldsymbol{\xi}$ in $L^{1}(\mathbb{T}$ ) (called a spectral shift function for the pair $\left.\left\{T_{1}, T_{0}\right\}\right)$ such that the following trace formula

$$
\operatorname{trace}\left(f\left(T_{1}\right)-f\left(T_{0}\right)\right)=\int_{\mathbb{T}} f^{\prime}(\zeta) \boldsymbol{\xi}(\zeta) d \zeta
$$

holds for an arbitrary operator Lipschitz function $f$ analytic in $\mathbb{D}$.

To obtain the main result, we combine two approaches. The first approach is based on double operator integrals with respect to semi-spectral measures and uses an idea of 
[BS2]. It leads to a trace formula trace $(f(T)-f(R))=\int_{\mathbb{T}} f^{\prime}(\zeta) d \nu(\zeta)$ for an arbitrary operator Lipschitz function $f$ analytic in $\mathbb{D}$, where $\nu$ is a Borel measure on $\mathbb{T}$.

The second approach develops ideas of Krein [Kr1] - [Kr4] and relies on the study of the perturbation determinant for pairs $\left\{L_{1}, L_{0}\right\}$ of m-accumulative operators and allows us to prove the trace formula for resolvents without additional restrictions on $\left\{L_{1}, L_{0}\right\}$ imposed in [MN2].

We denote by $\mathcal{B}(\mathscr{H})$ the set of bounded linear operators on a Hilbert space $\mathscr{H}$ and by $\boldsymbol{S}_{p}$ the Schatten-von Neumann ideal. In particular, $\boldsymbol{S}_{1}$ is the trace class. For a closed densely defined operator $Q$, we use the notation $\rho(Q), \sigma_{\mathrm{p}}(Q)$, and $\sigma_{\mathrm{c}}(Q)$ denote the resolvent set and the point and the continuous spectra of $Q$. Recall that $\sigma_{\mathrm{c}}(Q)=\{\lambda \notin$ $\left.\sigma_{\mathrm{p}}(Q): \operatorname{Range}(Q-\lambda I) \neq \operatorname{clos} \operatorname{Range}(Q-\lambda I)=\mathscr{H}\right\}$.

\section{Double operator integrals and Schur multipliers}

Double operator integrals

$$
\iint \Phi(x, y) d E_{1}(x) Q d E_{2}(y)
$$

were introduced by Yu.L. Daletskii and S.G. Krein in [DK]. Later Birman and Solomyak elaborated their beautiful theory of double oparator integrals [BS1] (see also [AP1] and references there). Here $\Phi$ is a bounded measurable function, $E_{1}$ and $E_{2}$ are spectral measures on Hilbert space, and $Q$ is a bounded linear operator. Such double operator integrals are defined for arbitrary bounded measurable functions $\Phi$ if $Q$ is a HilbertSchmidt operator. If $Q$ is an arbitrary bounded operator, then for the double operator integral to make sense, $\Phi$ has to be a Schur multiplier with respect to $E_{1}$ and $E_{2}$, (see $[\mathrm{Pe} 1]$ and [AP1]). It is well known (see [Pe1] and [AP1]) that $\Phi$ is a Schur multiplier if and only if it admits a representation

$$
\Phi(x, y)=\sum_{n \geq 0} \varphi_{n}(x) \psi_{n}(y)
$$

where the $\varphi_{n}$ and $\psi_{n}$ are measurable functions such that

$$
\sum_{n \geq 0}\left|\varphi_{n}\right|^{2} \in L_{E_{1}}^{\infty} \quad \text { and } \quad \sum_{n \geq 0}\left|\psi_{n}\right|^{2} \in L_{E_{2}}^{\infty} .
$$

In this paper we deal with double operator integrals with respect to semi-spectral measures

$$
\iint \Phi(x, y) d \mathscr{E}_{1}(x) Q d \mathscr{E}_{2}(y)
$$

Such double operator integrals were introduced in [Pe3] (see also [Pe5]). We refer the reader to the recent survey [AP1] for detailed information.

If $T$ is a contraction on a Hilbert space $\mathscr{H}$, it has a minimal unitary dilation $U$, i.e., $U$ is a unitary operator on a Hilbert space $\mathscr{K}, \mathscr{K} \supset \mathscr{H}, T^{n}=P_{\mathscr{H}} U^{n} \mid \mathscr{H}$ for $n \geq 0$ and $\mathscr{K}$ is the closed linear span of $U^{n} \mathscr{H}, n \in \mathbb{Z}$ (see $[\mathrm{SNF}]$ ). Here $P_{\mathscr{H}}$ is the orthogonal projection onto $\mathscr{H}$. The semi-spectral measure $\mathscr{E}_{T}$ of $T$ is defined by

$$
\mathscr{E}_{T}(\Delta) \stackrel{\text { def }}{=} \underset{3}{\mathscr{H}} E_{U}(\Delta) \mid \mathscr{H}
$$


where $E_{U}$ is the spectral measure of $U$ and $\Delta$ is a Borel subset of $\mathbb{T}$. It is easy to see that $T^{n}=\int_{\mathbb{T}} \zeta^{n} d \mathscr{E}_{T}(\zeta), n \geq 0$.

\section{The role of divided differences}

A function $f$ in the disk-algebra $\mathrm{C}_{\mathrm{A}}$ is called operator Lipschitz if

$$
\|f(T)-f(R)\| \leq \text { const }\|T-R\|
$$

for all contractions $T$ and $R$. We use the notation $\mathrm{OL}_{\mathrm{A}}$ for the class of operator Lipschitz functions in $\mathrm{C}_{\mathrm{A}}$. For $f \in \mathrm{OL}_{\mathrm{A}}$, we denote by $\mathfrak{D} f$ the divided difference defined by

$$
(\mathfrak{D} f)(\zeta, \tau)=\frac{f(\zeta)-f(\tau)}{\zeta-\tau} \quad \text { for } \quad \zeta \neq \tau \quad \text { and } \quad(\mathfrak{D} f)(\zeta, \tau)=f^{\prime}(\zeta) \quad \text { for } \quad \zeta=\tau \text {. }
$$

Recall that by a theorem of Johnson and Williams [JW], operator Lipschitz functions must be differentiable everywhere on $\mathbb{T}$.

We are going to use the following representation of the divided difference $\mathfrak{D} f$ for functions in $\mathrm{OL}_{\mathrm{A}}$ (see [AP1], Theorems 3.9.1 and 3.9.2):

Let $f$ be a function analytic in $\mathbb{D}$. Then $f \in \mathrm{OL}_{\mathrm{A}}$ if and only if $f$ admits a representation

$$
(\mathfrak{D} f)(z, w)=\sum_{n \geq 1} \varphi_{n}(z) \psi_{n}(w), \quad z, w \in \mathbb{D}
$$

where $\varphi_{n}$ and $\psi_{n}$ are functions in $\mathrm{C}_{\mathrm{A}}$ such that

$$
\left(\sup _{z \in \mathbb{D}} \sum_{n \geq 1}\left|\varphi_{n}(z)\right|^{2}\right)\left(\sup _{w \in \mathbb{D}} \sum_{n \geq 1}\left|\psi_{n}(w)\right|^{2}\right)<\infty
$$

If $f \in \mathrm{OL}_{\mathrm{A}}$, then the functions $\varphi_{n}$ and $\psi_{n}$ can be chosen so that the left-hand side of (3.2) is equal to $\|f\|_{\mathrm{OL}_{\mathrm{A}}}$.

Let $T_{0}$ and $T_{1}$ be contractions on Hilbert space, and let $\mathscr{E}_{0}$ and $\mathscr{E}_{1}$ be their semispectral measures. Suppose now that $f \in \mathrm{OL}_{\mathrm{A}}$. Consider a representation of $\mathfrak{D} f$ in the

form (3.1), where $\varphi_{n}$ and $\psi_{n}$ are functions in $\mathrm{C}_{\mathrm{A}}$ satisfying (3.2). Then for a bounded linear operator $K$, we have

$$
\iint_{\mathbb{T} \times \mathbb{T}}(\mathfrak{D} f)(\zeta, \tau) d \mathscr{E}_{1}(\zeta) K d \mathscr{E}_{0}(\tau)=\sum_{n=1}^{\infty} \varphi_{n}\left(T_{1}\right) K \psi_{n}\left(T_{0}\right)
$$

(see Section 3.9 of [AP1]). This implies (see Theorem 3.9 .9 of [AP1]) that

$$
f\left(T_{1}\right)-f\left(T_{0}\right)=\iint_{\mathbb{T} \times \mathbb{T}}(\mathfrak{D} f)(\zeta, \tau) d \mathscr{E}_{1}(\zeta)\left(T_{1}-T_{0}\right) d \mathscr{E}_{0}(\tau)
$$

\section{Differentiation in the strong operator topology}

The following theorem is a generalization of Theorem 3.5.6 of [AP1] and Theorem 3.1 of $[\mathrm{AP} 2]$. 
Theorem 4.1. Let $f \in \mathrm{OL}_{\mathrm{A}}$ and let $T_{0}$ and $T_{1}$ be contractions on Hilbert space and $T_{t} \stackrel{\text { def }}{=} T_{0}+t\left(T_{1}-T_{0}\right), 0 \leq t \leq 1$. Then

$$
\lim _{s \rightarrow 0} \frac{1}{s}\left(f\left(T_{t+s}\right)-f\left(T_{t}\right)\right)=\iint_{\mathbb{T} \times \mathbb{T}}(\mathfrak{D} f)(\zeta, \tau) d \mathscr{E}_{t}(\zeta)\left(T_{1}-T_{0}\right) d \mathscr{E}_{t}(\tau)
$$

in the strong operator topology, where $\mathscr{E}_{t}$ is the semi-spectral measure of $T_{t}$.

We need the following elementary fact:

Lemma 3.5.9 of [AP1]. Let $\left\{X_{n}\right\}_{n \geq 1}$ be a sequence of bounded linear operators on a Hilbert space $\mathscr{H}$ and let $\left\{u_{n}\right\}_{n \geq 1}$ be a sequence of vectors in $\mathscr{H}$. Suppose that

$$
\sum_{n \geq 1} X_{n} X_{n}^{*} \leq a^{2} I \quad \text { and } \quad \sum_{n \geq 1}\left\|u_{n}\right\|^{2} \leq b^{2}
$$

for $a, b \geq 0$. Then the series $\sum_{n \geq 1} X_{n} u_{n}$ converges weakly and $\left\|\sum_{n \geq 0} X_{n} u_{n}\right\| \leq a b$.

Proof of Theorem 4.1. Let $\varphi_{n}$ and $\psi_{n}$ are functions in $\mathrm{C}_{\mathrm{A}}$ that satisfy (3.1) and (3.2). It follows from (3.4) and (3.3) that it suffices to prove that

$$
\lim _{s \rightarrow 0} \sum_{n \geq 1} \varphi_{n}\left(T_{t+s}\right)\left(T_{1}-T_{0}\right) \psi_{n}\left(T_{t}\right)=\sum_{n \geq 1} \varphi_{n}\left(T_{t}\right)\left(T_{1}-T_{0}\right) \psi_{n}\left(T_{t}\right) .
$$

Note that the limit should be taken in the strong operator topology while the series converge in the weak operator topology.

Let $u$ be a vector of the Hilbert space. We have to prove that

$$
\lim _{s \rightarrow 0}\left(\varphi_{n}\left(T_{t+s}\right)-\varphi_{n}\left(T_{t}\right)\right)\left(T_{1}-T_{0}\right) \psi_{n}\left(T_{t}\right) u=\mathbf{0}
$$

in the Hilbert space norm.

We may assume that $\|u\|=1,\|f\|_{\mathrm{OL}_{\mathrm{A}}} \leq 1, \sum_{n \geq 1}\left|\varphi_{n}\right|^{2} \leq 1$ and $\sum_{n \geq 1}\left|\psi_{n}\right|^{2} \leq 1$.

Put $u_{n} \stackrel{\text { def }}{=}\left(T_{1}-T_{0}\right) \psi_{n}\left(T_{t}\right) u$. We have

$$
\sum_{n \geq 1}\left\|u_{n}\right\|^{2} \leq\left\|T_{1}-T_{0}\right\|^{2} \sum_{n \geq 1}\left\|\psi_{n}\left(T_{t}\right) u\right\|^{2}
$$

Suppose now that $U$ is a unitary dilation of $T_{t}$. We have

$$
\sum_{n \geq 1}\left\|\psi_{n}\left(T_{t}\right) u\right\|^{2} \leq \sum_{n \geq 1}\left\|\psi_{n}(U) u\right\|^{2}=\sum_{n \geq 1}\left(\left|\psi_{n}\right|^{2}(U) u, u\right)^{2} \leq 1
$$

and so $\sum_{n \geq 1}\left\|u_{n}\right\|^{2} \leq\left\|T_{1}-T_{0}\right\|^{2}$. Let $\varepsilon>0$. We can select a positive integer $N$ such that $\sum_{n>N}\left\|u_{n}\right\|^{2}<\varepsilon^{2}$. Let us show that

$$
\sum_{n \geq 1} \varphi_{n}\left(T_{t}\right)\left(\varphi_{n}\left(T_{t}\right)\right)^{*} \leq I \quad \text { and } \sum_{n \geq 1} \varphi_{n}\left(T_{t+s}\right)\left(\varphi_{n}\left(T_{t+s}\right)\right)^{*} \leq I .
$$

Indeed, let $U$ be a unitary dilation of $T_{t}$ on a Hilbert space $\mathscr{K}$ that contains $\mathscr{H}$. It is easy to verify that $\left\|\left(\varphi_{n}\left(T_{t}\right)\right)^{*} v\right\|_{\mathscr{H}} \leq\left\|\left(\varphi_{n}(U)\right)^{*} v\right\|_{\mathscr{K}}$. We have

$$
\sum_{n \geq 1}\left(\varphi_{n}\left(T_{t}\right)\left(\varphi_{n}\left(T_{t}\right)\right)^{*} v, v\right)=\sum_{n \geq 1}\left\|\left(\varphi_{n}\left(T_{t}\right)\right)^{*} v\right\|_{\mathscr{H}}^{2} \leq \sum_{n \geq 1}\left\|\left(\varphi_{n}(U)\right)^{*} v\right\|_{\mathscr{H}}^{2} \leq\|v\|_{\mathscr{H}}^{2}
$$


because $\sum_{n \geq 1}\left\|\varphi_{n}\right\|^{2} \leq 1$. This means that $\sum_{n \geq 1} \varphi_{n}\left(T_{t}\right)\left(\varphi_{n}\left(T_{t}\right)\right)^{*} \leq I$. In the same way we can prove that $\sum_{n \geq 1} \varphi_{n}\left(T_{t+s}\right)\left(\varphi_{n}\left(T_{t+s}\right)\right)^{*} \leq I$.

It follows from the above lemma that for all $t$,

$$
\left\|\sum_{n \geq 1}\left(\varphi_{n}\left(T_{t+s}\right)-\varphi_{n}\left(T_{t}\right)\right) u_{n}\right\| \leq 2 \varepsilon .
$$

It is easy to see that $\left\|\varphi_{n}\left(T_{t+s}\right)-\varphi_{n}\left(T_{t}\right)\right\| \rightarrow 0 \quad$ as $\quad s \rightarrow 0$ (it suffices to approximate $\varphi_{n}$ be analytic polynomials). Thus

$$
\left\|\sum_{n=1}^{N}\left(\varphi_{n}\left(T_{t+s}\right)-\varphi_{n}\left(T_{t}\right)\right) u_{n}\right\| \leq\left\|T_{1}-T_{0}\right\| \sum_{n=1}^{N}\left\|\varphi_{n}\left(T_{t+s}\right)-\varphi_{n}\left(T_{t}\right)\right\|<\varepsilon
$$

if $s$ is sufficiently close to 0 . Thus $\left\|\sum_{n \geq 1}\left(\varphi_{n}\left(T_{t+s}\right)-\varphi_{n}\left(T_{t}\right)\right) u_{n}\right\|<3 \varepsilon$ for $s$ sufficiently close to 0 which completes the proof.

\section{A trace formula for double operator integrals}

Theorem 5.1. Let $f \in \mathrm{OL}_{\mathrm{A}}$. Suppose that $T$ is a contraction with semi-spectral measure $\mathscr{E}$ and $K$ is a trace class operator on Hilbert space. Then

$$
\operatorname{trace}\left(\iint_{\mathbb{T} \times \mathbb{T}}(\mathfrak{D} f)(\zeta, \tau) d \mathscr{E}(\zeta) K d \mathscr{E}(\tau)\right)=\int_{\mathbb{T}} f^{\prime}(\zeta) d \mu(\zeta),
$$

where $\mu$ is a complex Borel measure on $\mathbb{T}$ defined by $\mu(\Delta)=\operatorname{trace}(K \mathscr{E}(\Delta))$.

Proof. Let $\varphi_{n}$ and $\psi_{n}$ be functions in $\mathrm{C}_{\mathrm{A}}$ satisfying (3.1) and (3.2). By (3.3),

$$
\iint_{\mathbb{T} \times \mathbb{T}}(\mathfrak{D} f)(\zeta, \tau) d \mathscr{E}(\zeta) K d \mathscr{E}(\tau)=\sum_{n \geq 1} \varphi_{n}(T) K \psi_{n}(T),
$$

and so

$$
\begin{aligned}
\operatorname{trace}\left(\iint_{\mathbb{T} \times \mathbb{T}}(\mathfrak{D} f)(\zeta, \tau) d \mathscr{E}(\zeta) K d \mathscr{E}(\tau)\right)=\sum_{n \geq 1} \operatorname{trace}\left(\varphi_{n}(T) K \psi_{n}(T)\right) \\
=\sum_{n \geq 1} \operatorname{trace}\left(K\left(\varphi_{n} \psi_{n}\right)(T)\right)=\operatorname{trace}\left(K \int_{\mathbb{T}}\left(\sum_{n \geq 1} \varphi_{n} \psi_{n}\right)(\zeta) d \mathscr{E}(\zeta)\right) \\
=\operatorname{trace}\left(K \int_{\mathbb{T}}(\mathfrak{D} f)(\zeta, \zeta) d \mathscr{E}(\zeta)\right)=\operatorname{trace}\left(K \int_{\mathbb{T}} f^{\prime}(\zeta) d \mathscr{E}(\zeta)\right)=\int_{\mathbb{T}} f^{\prime}(\zeta) d \mu(\zeta) .
\end{aligned}
$$

This completes the proof.

\section{A trace formula for functions of contractions}

Theorem 6.1. Let $T_{0}$ and $T_{1}$ be contractions on Hilbert space such that $T_{1}-T_{0} \in \boldsymbol{S}_{1}$. Then there exists a complex Borel measure $\nu$ on $\mathbb{T}$ such that the trace formula

$$
\operatorname{trace}\left(f\left(T_{1}\right)-f\left(T_{0}\right)\right)=\int_{\mathbb{T}} f^{\prime}(\zeta) d \nu(\zeta)
$$


holds for every $f$ in $\mathrm{OL}_{\mathrm{A}}$.

Proof. Let $K \stackrel{\text { def }}{=} T_{1}-T_{0}$. Consider the family of contractions $T_{t} \stackrel{\text { def }}{=} T+t K, 0 \leq t \leq 1$. By Theorem 4.1, the function $t \mapsto f\left(T_{t}\right)$ is differentiable in the strong operator topology. Put

$$
Q_{t} \stackrel{\text { def }}{=} \lim _{s \rightarrow 0} \frac{1}{s} \iint_{\mathbb{T} \times \mathbb{T}}(\mathfrak{D} f)(\zeta, \tau) d \mathscr{E}_{t}(\zeta) K d \mathscr{E}_{t}(\tau) .
$$

Since $K \in \boldsymbol{S}_{1}$ and $f \in \mathrm{OL}_{\mathrm{A}}$, we have

$$
Q_{t} \in \boldsymbol{S}_{1}, 0 \leq t \leq 1, \quad \text { and } \sup _{t \in[0,1]}\left\|Q_{t}\right\|_{\boldsymbol{S}_{1}}<\infty .
$$

It follows from the definition of $Q_{t}$ that the function $t \mapsto Q_{t} u$ is measurable for every vector $u$. Then the function $t \mapsto \operatorname{trace}\left(Q_{t} W\right)$ is measurable for an arbitrary bounded operator $W$. In other words the $\boldsymbol{S}_{1}$-valued function $t \mapsto Q_{t}$ is weakly measurable. Then it must be strongly measurable because $\boldsymbol{S}_{1}$ is separable (see [Yo], Ch. V, § 4) and

$$
f\left(T_{1}\right)-f\left(T_{0}\right)=\int_{0}^{1} Q_{t} d t
$$

where the integral is understood in the sense of Bochner.

By Theorem 5.1,

$$
\operatorname{trace} Q_{t}=\int_{\mathbb{T}} f^{\prime}(\zeta) d \nu_{t}(\zeta)
$$

where $\nu_{t}$ is the complex Borel measure on $\mathbb{T}$ defined by $\nu_{t}(\Delta) \stackrel{\text { def }}{=} \operatorname{trace}\left(K \mathscr{E}_{t}(\Delta)\right)$ for a Borel subset $\Delta$ of $\mathbb{T}$. It would be tempting to define the measure $\nu$ by

$$
\nu(\Delta)=\int_{0}^{1} \nu_{t}(\Delta) d t
$$

and conclude that formula (6.1) holds for this measure $\nu$. However, it is not quite clear why the function $t \mapsto \nu_{t}(\Delta)$ is measurable.

To overcome this problem, we consider the dual space to the disk-algebra $\mathrm{C}_{\mathrm{A}}$. It can naturally be identified with the quotient space $\mathcal{M} / H^{1}$, where $\mathcal{M}$ is the space of complex Borel measures on $\mathbb{T}$ and $H^{1}$ is the Hardy class. Consider the function $t \mapsto \dot{\nu}_{t}$, where $\dot{\nu}_{t}$ is the coset in $\mathcal{M} / H^{1}$ that corresponds to $\nu_{t}$. Let us show that the map $t \mapsto \dot{\nu}_{t}$ is continuous in the weak-* topology on $\mathcal{M} / H^{1}$. Indeed, let $h \in \mathrm{C}_{\mathrm{A}}$. We have $\left\langle h, \dot{\nu}_{t}\right\rangle=\operatorname{trace}\left(K h\left(T_{t}\right)\right)$. The result follows from the fact that the map $t \mapsto h\left(T_{t}\right)$ is continuous in the operator norm. This can be proved easily by approximating $h$ by analytic polynomials.

Consider now the integral $\int_{0}^{1} \dot{\nu}_{t} d t$. It is an element of $\mathcal{M} / H^{1}$, and so it is equal to $\dot{\nu}$ for some $\nu \in \mathcal{M}$. It remains to observe that $\nu$ satisfies equality (6.1).

\section{The existence of spectral shift functions for a pair of contractions}

Definition. A densely defined operator $L$ in $\mathscr{H}$ is called dissipative if $\operatorname{Im}(L x, x) \geq 0$ for $x$ in the domain $\operatorname{dom}(L)$ of $L$. It is called maximal dissipative (or $m$-dissipative) if $L$ has no proper dissipative extension. An operator $L$ is called accumulative (maccumulative) if $-L$ is dissipative (m-dissipative). 
First, we present an improvement of Theorem 3.14 of [MN2] for pairs $\left\{L_{1}, L_{0}\right\}$ of m-dissipative operators.

Theorem 7.1. Let $L_{1}$ and $L_{0}$ be m-dissipative operators such that $\left(\rho\left(L_{0}\right) \cup \sigma_{\mathrm{c}}\left(L_{0}\right)\right) \cap$ $\mathbb{C}_{+} \neq \varnothing$. Suppose that $\left(L_{1}+\mathrm{i} I\right)^{-1}-\left(L_{0}+\mathrm{i} I\right)^{-1} \in \boldsymbol{S}_{1}$, i.e., $L_{1}$ and $L_{0}$ are resolvent comparable. Then there exists a complex-valued function $\boldsymbol{\omega} \in L^{1}\left(\mathbb{R},\left(1+t^{2}\right)^{-1}\right)$ (a spectral shift function for the pair $\left.\left\{L_{1}, L_{0}\right\}\right)$ such that the following trace formula holds

$$
\operatorname{trace}\left(\left(L_{1}-\tau I\right)^{-1}-\left(L_{0}-\tau I\right)^{-1}\right)=-\int_{\mathbb{R}} \frac{\boldsymbol{\omega}(t)}{(t-\tau)^{2}} d t, \quad \tau \in \mathbb{C}_{-} .
$$

If $L_{0}=L_{0}^{*}$ (respectively, $\left.L_{1}=L_{1}^{*}\right)$, then one can select $\boldsymbol{\omega}$ such that $\operatorname{Im}(\boldsymbol{\omega}(t)) \geq 0$ (respectively, $\operatorname{Im}(\boldsymbol{\omega}(t)) \leq 0)$ a.e. on $\mathbb{R}$.

The proof of Theorem 7.1 is given in the Appendix. In what follows we use the notation $\mathscr{S}\left\{L_{1}, L_{0}\right\}$ for the set of spectral shift functions for $\left\{L_{1}, L_{0}\right\}$.

Theorem 7.2. Let $T_{1}$ and $T_{0}$ be contractions with $T_{1}-T_{0} \in \boldsymbol{S}_{1}$. Then there is a complex-valued function $\boldsymbol{\xi}$ in $L^{1}(\mathbb{T})$ (a spectral shift function for $\left\{T_{1}, T_{0}\right\}$ ) such that

$$
\operatorname{trace}\left(\left(T_{1}-\lambda I\right)^{-1}-\left(T_{0}-\lambda I\right)^{-1}\right)=-\int_{\mathbb{T}} \frac{\boldsymbol{\xi}(\zeta)}{(\zeta-\lambda)^{2}} d \zeta, \quad|\lambda|>1 .
$$

If $T_{0}$ (respectively, $T_{1}$ ) is unitary, we can select $\boldsymbol{\xi}$ such that $\operatorname{Im}(\boldsymbol{\xi}(\zeta)) \leq 0$ (respectively, $\operatorname{Im}(\boldsymbol{\xi}(\zeta)) \geq 0)$ for almost all $\zeta \in \mathbb{T}$.

Proof. (i). Let us first assume that $\operatorname{Ker}\left(T_{1}+I\right)=\operatorname{Ker}\left(T_{0}+I\right)=\{0\}$ and $0 \in$ $\rho\left(T_{0}\right) \cup \sigma_{c}\left(T_{0}\right)$. Note that for a contraction $T$, the condition $\operatorname{Ker}(T+I)=\{0\}$ is equivalent to $\operatorname{Ker}\left(T^{*}+I\right)=\{0\}$. Therefore the operators

$$
L_{1}=-\mathrm{i} I+2 \mathrm{i}\left(I+T_{1}\right)^{-1} \quad \text { and } \quad L_{0}=-\mathrm{i} I+2 \mathrm{i}\left(I+T_{0}\right)^{-1}
$$

exist and are densely defined. One can easily verify that $L_{1}$ and $L_{0}$ are dissipative. Since the operators $L_{1}+\mathrm{i} I$ and $L_{0}+\mathrm{i} I$ are onto, they are m-dissipative. Let us show that $L_{1}$ and $L_{0}$ satisfy the hypotheses of Theorem 7.1.

Clearly, the map $\lambda \mapsto \tau \stackrel{\text { def }}{=} \mathrm{i}(1-\lambda)(1+\lambda)^{-1}$ maps $\mathbb{D}$ onto $\mathbb{C}_{+}$and maps $\mathbb{C} \backslash \operatorname{clos} \mathbb{D}$ onto $\mathbb{C}_{-}$. In accordance with (7.3),

$$
\begin{aligned}
& \left(L_{1}-\tau I\right)^{-1}=-\frac{1+\lambda}{2 \mathrm{i}} I-\frac{(1+\lambda)^{2}}{2 \mathrm{i}}\left(T_{1}-\lambda I\right)^{-1}, \\
& \left(L_{0}-\tau I\right)^{-1}=-\frac{1+\lambda}{2 \mathrm{i}} I-\frac{(1+\lambda)^{2}}{2 \mathrm{i}}\left(T_{0}-\lambda I\right)^{-1},
\end{aligned}
$$

where $\tau \in \mathbb{C}_{-}$. It follows that $L_{1}$ and $L_{0}$ are resolvent comparable.

Let us show that $\mathrm{i} \in \rho\left(L_{0}\right) \cup \sigma_{\mathrm{c}}\left(L_{0}\right)$. By (7.3), $T_{0}=\left(\mathrm{i} I-L_{0}\right)\left(\mathrm{i} I+L_{0}\right)^{-1}$, and so the condition $\operatorname{Ker} T_{0}=\{\mathbf{0}\}$ is equivalent to $\operatorname{Ker}\left(L_{0}-\mathrm{i} I\right)=\{\mathbf{0}\}$. Similarly, $T_{0}$ has dense range if and only if $L_{0}-\mathrm{i} I$ has dense range. This proves that $\mathrm{i} \in \rho\left(L_{0}\right) \cup \sigma_{\mathrm{c}}\left(L_{0}\right)$. By Theorem 7.1, there exists $\boldsymbol{\omega} \in L^{1}\left(\mathbb{R},\left(1+t^{2}\right)^{-1}\right)$ such that trace formula (7.1) holds. Combining this formula with (7.4) and setting

$$
t \stackrel{\text { def }}{=} \mathrm{i}(1-\zeta)(1+\zeta)^{-1}=\mathrm{i}\left(1-e^{i \theta}\right)\left(1+e_{8}^{i \theta}\right)^{-1} \in \mathbb{R}, \quad \zeta=e^{i \theta} \in \mathbb{T}, \quad \theta \in(-\pi, \pi],
$$


and $\boldsymbol{\xi}(\zeta) \stackrel{\text { def }}{=} \boldsymbol{\omega}\left(\mathrm{i}(1-\zeta)(1+\zeta)^{-1}\right)$, i.e.,

$$
\boldsymbol{\xi}\left(e^{i \theta}\right)=\boldsymbol{\omega}\left(\mathrm{i}\left(1-e^{i \theta}\right)\left(1+e^{\mathrm{i} \theta}\right)^{-1}\right)=\boldsymbol{\omega}(\tan (\theta / 2)), \quad \theta \in(-\pi, \pi],
$$

we obtain

$$
\begin{gathered}
-\frac{(1+\lambda)^{2}}{2 i} \operatorname{trace}\left(\left(T_{1}-\lambda I\right)^{-1}-\left(T_{0}-\lambda I\right)^{-1}\right)=\operatorname{trace}\left(\left(L_{1}-\tau I\right)^{-1}-\left(L_{0}-\tau I\right)^{-1}\right) \\
=-\int_{\mathbb{R}} \frac{\boldsymbol{\omega}(t)}{(t-\tau)^{2}} d t=\frac{(1+\lambda)^{2}}{2 \mathrm{i}} \int_{\mathbb{T}} \frac{\boldsymbol{\xi}(\zeta)}{(\zeta-\lambda)^{2}} d \zeta, \quad|\lambda|>1 .
\end{gathered}
$$

This proves (7.2). Moreover, $\int_{-\pi}^{\pi}\left|\boldsymbol{\xi}\left(e^{\mathrm{i} \theta}\right)\right| d \theta=2 \int_{\mathbb{R}}|\boldsymbol{\omega}(t)|\left(1+t^{2}\right)^{-1} d t<\infty$, i.e., $\boldsymbol{\xi} \in L^{1}(\mathbb{T})$.

(ii). Assume now that at least one of the conditions $\operatorname{Ker}\left(T_{1}-I\right)=\{\boldsymbol{0}\}$ and $\operatorname{Ker}\left(T_{0}-I\right)=\{\mathbf{0}\}$ is violated. We construct an auxiliary pair $\left\{\widehat{T}_{1}, \widehat{T}_{0}\right\}$ of contractions with $\operatorname{Ker}\left(\widehat{T}_{1}-I\right)=\operatorname{Ker}\left(\widehat{T}_{0}-I\right)=\{\mathbf{0}\}$.

First, we observe that for each eigenvalue $\zeta \in \sigma_{\mathrm{p}}(T),|\zeta|=1$, the eigensubspace $\operatorname{Ker}(T-\zeta I)$ is also the eigensubspace of the minimal unitary dilation $U$, i.e., $\operatorname{Ker}(U-$ $\zeta I)=\operatorname{Ker}(T-\zeta I)($ see $[\mathrm{SNF}])$. Therefore the set $\sigma_{\mathrm{p}}(T) \cap \mathbb{T}$ is at most countable. This implies the existence of a number $\varkappa \in \mathbb{T}$ such that the contractions $\widehat{T} \stackrel{\text { def }}{=} \varkappa T$ and $\widehat{T}_{0} \stackrel{\text { def }}{=} \varkappa T_{0}$ have the required properties

$$
\operatorname{Ker}(\widehat{T}+I)=\operatorname{Ker}\left(\widehat{T}^{*}+I\right)=\{0\} \quad \text { and } \quad \operatorname{Ker}\left(\widehat{T}_{0}+I\right)=\operatorname{Ker}\left(\widehat{T}_{0}^{*}+I\right)=\{0\} .
$$

In accordance with (i), there exists a function $\widehat{\boldsymbol{\xi}} \in L^{1}(\mathbb{T})$ such that

$$
\operatorname{trace}\left((\widehat{T}-\hat{\lambda} I)^{-1}-\left(\widehat{T}_{0}-\hat{\lambda} I\right)^{-1}\right)=-\int_{\mathbb{T}} \widehat{\boldsymbol{\xi}}(\hat{\zeta})(\hat{\zeta}-\hat{\lambda})^{-2} d \hat{\zeta}, \quad|\hat{\lambda}|>1 .
$$

Setting $\lambda=\bar{\varkappa} \hat{\lambda}, \zeta=\bar{\varkappa} \hat{\zeta}$ and $\boldsymbol{\xi}(\zeta)=\widehat{\boldsymbol{\xi}}(\varkappa \zeta)$, we obtain (7.2).

(iii). Suppose now that the condition $0 \in \rho\left(T_{0}\right) \cup \sigma_{\mathrm{c}}\left(T_{0}\right)$ is violated, i.e., $0 \in \sigma_{\mathrm{p}}\left(T_{0}\right) \cup$ $\sigma_{\mathrm{p}}\left(T_{0}^{*}\right)$. Let us first assume that $0 \in \sigma_{\mathrm{p}}\left(T_{0}\right)$. Consider the Hilbert space $\mathscr{H} \oplus \mathscr{K}$, where $\mathscr{H}$ is the space, on which $T_{0}$ and $T_{1}$ are defined and $\mathscr{K}$ is an infinite-dimensional Hilbert space. Consider the operators

$$
\check{T}_{1}=T_{1} \oplus \mathbf{0} \quad \text { and } \quad \check{T}_{0}=T_{0} \oplus \mathbf{0} .
$$

on this space. Clearly, $\check{T}_{1}-\check{T}_{0} \in \boldsymbol{S}_{1}$. Let $T_{0}=V_{0}\left|T_{0}\right|$ be the polar decompostion of $T_{0}$, where $V_{0}$ is a partial isometry that maps clos Range $T_{0}^{*}=\mathscr{H} \ominus \operatorname{Ker} T_{0}$ onto clos Range $T_{0}$.

We have $\operatorname{Ker} \check{T}_{0}=\operatorname{Ker} T_{0} \oplus \mathscr{K}$ and $\left(\text { Range } \check{T}_{0}\right)^{\perp}=\left(\text { Range } T_{0}\right)^{\perp} \oplus \mathscr{K}$. Note that $\operatorname{dim} \operatorname{Ker} \check{T}_{0}=\operatorname{dim}\left(\text { Range } \check{T}_{0}\right)^{\perp}=\infty$. Let $K$ : Ker $\check{T}_{0} \longrightarrow \operatorname{Ker} \check{T}_{0}$ be a trace class nonnegative self-adjoint contraction such that $\operatorname{Ker} K=\{\mathbf{0}\}$ and let $W$ be an isometry from Ker $\check{T}_{0}$ onto (Range $\left.\check{T}_{0}\right)^{\perp}$. Clearly, such an isometry exists. Put

$$
R f \stackrel{\text { def }}{=} \begin{cases}T_{0} f, & f \in \operatorname{Ker}\left(T_{0}\right)^{\perp}=\mathscr{H} \ominus \operatorname{Ker}\left(T_{0}\right), \\ W K f, & f \in \operatorname{Ker}\left(\check{T}_{0}\right)=\operatorname{Ker}\left(T_{0}\right) \oplus \mathscr{K} .\end{cases}
$$

Let $f \in \mathscr{H} \oplus \mathscr{K}$ and $f=f_{1}+f_{2}$, where $f_{1} \in\left(\operatorname{Ker} T_{0}\right)^{\perp}$ and $f_{2} \in \operatorname{Ker} \check{T}_{0}$. Then

$$
\begin{aligned}
& \|R f\|^{2}=\left\|T_{0} f_{1}+W K f_{2}\right\|^{2}=\left\|T_{0} f_{1}\right\|^{2}+2 \operatorname{Re}\left(T_{0} f_{1}, W K f_{2}\right)+\left\|W K f_{2}\right\|^{2}
\end{aligned}
$$

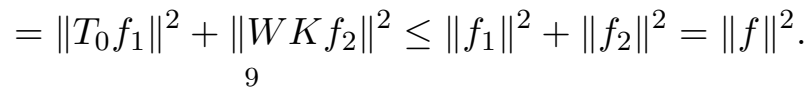


Thus, $R$ is a contraction on $\mathscr{H} \oplus \mathscr{K}$. Moreover, $\operatorname{Ker} R=\{\mathbf{0}\}$ because $\operatorname{Ker}\left(T_{0}\right)=$ $\operatorname{Ker}(W K)=\{\mathbf{0}\}$. It is easy to see that $R$ has dense range in $\mathscr{H} \oplus \mathscr{K}$. Hence, $0 \in \rho(R) \cup$ $\sigma_{\mathrm{c}}(R)$. Let $P_{\text {Ker } \check{T}_{0}}$ be the orthogonal projection onto the subspace Ker $\check{T}_{0}$. Combining (7.8) with (7.9) and keeping in mind that $K \in \boldsymbol{S}_{1}$, we find that $R-\check{T}_{0}=W K P_{\operatorname{Ker} \check{T}_{0}^{\prime}} \in \boldsymbol{S}_{1}$. It follows from what we have established in (ii) that there exists a spectral shift function $\boldsymbol{\xi}_{1}$ for the pair $\left\{\check{T}_{0}, R\right\}$, i.e., the identity

$$
\operatorname{trace}\left(\left(\check{T}_{0}-\lambda I\right)^{-1}-(R-\lambda I)^{-1}\right)=-\int_{\mathbb{T}} \boldsymbol{\xi}_{1}(\zeta)(\zeta-\lambda)^{-2} d \zeta, \quad|\lambda|>1,
$$

holds. Similarly,

$$
\left(\check{T}_{1}-R\right) f= \begin{cases}\left(T-T_{0}\right) f, & f \in\left(\operatorname{Ker} T_{0}\right)^{\perp}, \\ -W K f, & f \in \operatorname{Ker} \check{T}_{0} .\end{cases}
$$

Hence, $\check{T}_{1}-R \in \boldsymbol{S}_{1}$ and there exists a spectral shift function $\boldsymbol{\xi}_{2}$ for $\left\{\check{T}_{1}, R\right\}$ such that the following trace formula holds

$$
\operatorname{trace}\left(\left(\check{T}_{1}-\lambda I\right)^{-1}-(R-\lambda I)^{-1}\right)=-\int_{\mathbb{T}} \boldsymbol{\xi}_{2}(\zeta)(\zeta-\lambda)^{-2} d \zeta, \quad|\lambda|>1 .
$$

Combining (7.10) with (7.12) and setting $\boldsymbol{\xi}=\boldsymbol{\xi}_{2}-\boldsymbol{\xi}_{1}$, we obtain

$$
\operatorname{trace}\left(\left(\check{T}_{1}-\lambda I\right)^{-1}-\left(\check{T}_{0}-\lambda I\right)^{-1}\right)=-\int_{\mathbb{T}} \boldsymbol{\xi}(\zeta)(\zeta-\lambda)^{-2} d \zeta, \quad|\lambda|>1 .
$$

It follows from (7.8) that

$$
\begin{aligned}
\left(\check{T}_{1}-\lambda I\right)^{-1}-\left(\check{T}_{0}-\lambda I\right)^{-1} & =\left(T_{1}-\lambda I\right)^{-1} \oplus-\lambda^{-1} I-\left(T_{0}-\lambda I\right)^{-1} \oplus-\lambda^{-1} I \\
& =\left(\left(T_{1}-\lambda I\right)^{-1}-\left(T_{0}-\lambda I\right)^{-1}\right) \oplus \mathbf{0} .
\end{aligned}
$$

Finally, combining this identity with (7.13), we get trace formula (7.2).

Suppose now that $0 \in \sigma_{\mathrm{p}}\left(T_{0}^{*}\right) \backslash \sigma_{\mathrm{p}}\left(T_{0}\right)$. The above reasoning shows that identity (7.13) holds with $\left\{\check{T}_{1}, \check{T}_{0}\right\}$ and $\boldsymbol{\xi}$ replaced by $\left\{\check{T}_{1}^{*}, \check{T}_{0}^{*}\right\}$ and a function $\boldsymbol{\xi}_{*}$ in $\mathscr{S}\left\{\check{T}_{1}^{*}, \check{T}_{0}^{*}\right\}$. Setting $\boldsymbol{\xi} \stackrel{\text { def }}{=} \overline{\boldsymbol{\xi}}_{*}$ and taking to the complex conjugate in this identity, we arrive at (7.13).

(iv). Suppose now that $T_{0}$ is unitary. Then $0 \in \rho\left(T_{0}\right)$ and in accordance with (ii), there exists $\varkappa \in \mathbb{T}$ such that $-\bar{\varkappa} \notin \sigma_{p}\left(T_{0}\right) \cup \sigma_{p}(T)$. Therefore $L_{0}=\mathrm{i}\left(I-\varkappa T_{0}\right)\left(I+\varkappa T_{0}\right)^{-1}$ and $L_{1}=\mathrm{i}\left(I-\varkappa T_{1}\right)(I+\varkappa T)^{-1}$ are well defined. Moreover, $L_{0}=L_{0}^{*}, L_{1}$ is m-dissipative, and $\mathrm{i} \in \rho\left(H_{0}\right)$. By Theorem 7.1 there exists a function $\boldsymbol{\omega}$ in $\mathscr{S}\left\{L_{1}, L_{0}\right\}$ such that $\operatorname{Im} \boldsymbol{\omega} \geq 0$. On the other hand, in arcordance with (i), there is a spectral shift function $\widehat{\boldsymbol{\xi}}$ such that trace formula (7.7) holds, where $\widehat{T}=\varkappa T$ and $\widehat{T}_{0}=\varkappa T_{0}$. By (7.5), we get $\operatorname{Im} \widehat{\boldsymbol{\xi}} \geq 0$ on $\mathbb{T}$. Setting $\boldsymbol{\xi}(\zeta)=\widehat{\boldsymbol{\xi}}(\varkappa \zeta), \zeta \in \mathbb{T}$, we find that $\boldsymbol{\xi}$ is a spectral shift function for $\left\{T_{1}, T_{0}\right\}$ and $\operatorname{Im} \boldsymbol{\xi} \geq 0$ on $\mathbb{T}$. The case when $T_{1}$ unitary is treated similarly.

Another approach to trace formulae for the resolvents for a pair $\left\{T_{1}, T_{0}\right\}$ with unitary $T_{0}$ and a contraction $T_{1}$ satisfying $T_{1}-T_{0} \in \boldsymbol{S}_{1}$ and $D_{T_{1}} \stackrel{\text { def }}{=}\left(I-T_{1}^{*} T_{1}\right)^{1 / 2} \in \boldsymbol{S}_{1}$ was proposed by A. Rybkin [R2, R3, R4]. His formulae involve a complex spectral shift function, which is (A)-integrable but not Lebesgue integrable. 


\section{ReAl-VAlued SPECTRAL SHifT FunCTIONS}

We denote by $\mathscr{S}\left\{T_{1}, T_{0}\right\}$ the set of all spectral shift functions for the pair $\left\{T_{0}, T_{1}\right\}$. Let $\boldsymbol{\xi} \in \mathscr{S}\left\{T_{1}, T_{0}\right\}$. Clearly, an $L^{1}$ function $\boldsymbol{\xi}_{\natural}$ belongs to $\mathscr{S}\left\{T_{1}, T_{0}\right\}$ if and only if

$$
\boldsymbol{\xi}_{\natural}-\boldsymbol{\xi} \in H^{1}
$$

Theorem 8.1. Let $\left\{T_{1}, T_{0}\right\}$ be a pair of contractions satisfying $T_{1}-T_{0} \in \mathfrak{S}_{1}$ and let $\boldsymbol{\xi} \in \mathscr{S}\left\{T_{1}, T_{0}\right\}$. Suppose that

$$
(\operatorname{Im} \boldsymbol{\xi}) \log (1+|\operatorname{Im} \boldsymbol{\xi}|) \in L^{1}(\mathbb{T}) .
$$

Then there exists a real function in $\mathscr{S}\left\{T_{1}, T_{0}\right\}$.

If $\operatorname{Im} \boldsymbol{\xi} \geq 0$ for a.e. on $\mathbb{T}$ and there is a real function in $\mathscr{S}\left\{T_{1}, T_{0}\right\}$, then (8.2) holds.

Proof. For $g \in L^{1}(\mathbb{T})$, we put $\mathbb{P}_{+} g \stackrel{\text { def }}{=} \sum_{j \geq 0} \widehat{g}(j) z^{j}$ and $\mathbb{P}_{-} g \stackrel{\text { def }}{=} g-\mathbb{P}_{+} g$. We have

$$
\boldsymbol{\xi}=\operatorname{Re} \boldsymbol{\xi}+\mathrm{i} \operatorname{Im} \boldsymbol{\xi}=\operatorname{Re} \boldsymbol{\xi}+\mathrm{i} \mathbb{P}_{-}(\operatorname{Im} \boldsymbol{\xi})+i \mathbb{P}_{+}(\operatorname{Im} \boldsymbol{\xi}) .
$$

By Zygmund's theorem (see [Ko], Sect. V.C.3), inequality (8.2) implies that both $\mathbb{P}_{+}(\operatorname{Im} \boldsymbol{\xi})$ and $\mathbb{P}_{-}(\operatorname{Im} \boldsymbol{\xi})$ belong to $L^{1}(\mathbb{T})$. Put

$$
\boldsymbol{\xi}_{b} \stackrel{\text { def }}{=} \operatorname{Re} \boldsymbol{\xi}+\mathrm{i} \mathbb{P}_{-}(\operatorname{Im} \boldsymbol{\xi})-i \overline{\mathbb{P}_{-}(\operatorname{Im} \boldsymbol{\xi})} .
$$

Clearly, $\boldsymbol{\xi}_{b}$ is a real function in $L^{1}(\mathbb{T})$ and

$$
\boldsymbol{\xi}-\boldsymbol{\xi}_{b}=\mathrm{i}\left(\mathbb{P}_{+}(\operatorname{Im} \boldsymbol{\xi})+\overline{\mathbb{P}_{-}(\operatorname{Im} \xi)}\right) \in H^{1} .
$$

By $(8.1), \boldsymbol{\xi}_{b} \in \mathscr{S}\left\{T_{1}, T_{0}\right\}$.

Suppose now that $\boldsymbol{\xi}$ and $\boldsymbol{\xi}_{\sharp}$ belong to $\mathscr{S}\left\{T_{1}, T_{0}\right\}, \operatorname{Im} \boldsymbol{\xi} \geq 0$ and $\boldsymbol{\xi}_{\sharp}$ is real. Then by (8.1), $\boldsymbol{\xi}-\boldsymbol{\xi}_{\sharp} \in H^{1}$ and $\operatorname{Im}\left(\boldsymbol{\xi}-\boldsymbol{\xi}_{\sharp}\right)=\operatorname{Im} \boldsymbol{\xi} \geq 0$. It follows from M. Riesz' theorem (see [Ko], Sect. V.C.4) that (8.2) holds.

Theorem 8.2. Let $T_{0}$ and $T_{1}$ be contrarctions with trace class difference. Suppose that $\boldsymbol{\xi} \in \mathscr{S}\left\{T_{1}, T_{0}\right\}$ and $\operatorname{Im} \boldsymbol{\xi} \in L^{p}(\mathbb{T}, w)$ for a weight $w$ satisfying the Muckenhoupt condition $(\mathrm{A})_{p}, 1<p<\infty$. Then there is a real function $\boldsymbol{\xi}_{b} \in \mathscr{S}\left\{T_{1}, T_{0}\right\} \cap L^{p}(\mathbb{T}, w)$.

Proof. It follows easily from Hölder's inequality that $L^{p}(\mathbb{T}, w) \subset L^{1}(\mathbb{T})$. We can take the function $\boldsymbol{\xi}_{b}$ defined by (8.3), repeat the argument in the proof of Theorem 8.1 and use the Hunt-Muckenhoupt-Wheeden theorem (see [Ko]) instead of Zygmund's theorem.

Consider now the special case of pairs $\left\{L_{1}, L_{0}\right\}$, where $L_{0}=\mathbf{0}, L_{1}=L_{0}+\mathrm{i} V$ and $\mathbf{0} \leq V \in \boldsymbol{S}_{1}$. Clealy, $L_{1}$ is bounded, and so it is m-dissipative.

Theorem 8.3. Let $L_{0}=\mathbf{0}, L_{1}=L_{0}+\mathrm{i} V, \mathbf{0} \leq V \in \boldsymbol{S}_{1}$, and let $\left\{\alpha_{n}\right\}_{n \geq 0}$ be the sequence of eigenvalues of $V$ counted with multiplicities. Then

(i) The set $\mathscr{S}\left\{L_{1}, L_{0}\right\}$ contains a purely imaginary function;

(ii) The set $\mathscr{S}\left\{L_{1}, L_{0}\right\}$ contains a real-valued function if and only if

$$
\sum_{n \geq 0} \alpha_{n}\left|\log \alpha_{n}\right|<\infty
$$


Proof. (i) Consider the perturbation determinant $\Delta_{L_{1} / L_{0}}$ defined by

$$
\Delta_{L_{1} / L_{0}}(z)=\operatorname{det}\left(I+\mathrm{i} V\left(L_{0}-z I\right)^{-1}\right)=\operatorname{det}\left(I-\mathrm{i} z^{-1} V\right), \quad z \in \mathbb{C} \backslash\{0\} .
$$

The operator $V=V^{*}$ being of trace class, admits a spectral decomposition

$$
V=\sum_{n \geq 0} \alpha_{n} P_{n}=\sum_{n \geq 0} \alpha_{n}\left(\cdot, e_{n}\right) e_{n}, \quad \alpha_{n} \geq 1, \quad n \in \mathbb{N},
$$

where $\left\{e_{n}\right\}_{n=1}^{\infty}$ is an orthonormal basis, the orthogonal projection $P_{n}$ is defined by $P_{n}=$ $\left(\cdot, e_{n}\right) e_{n}$ is , and $C_{0} \stackrel{\text { def }}{=} \sum_{n=1}^{\infty} \alpha_{n}<\infty$. Setting $V_{n}=\alpha_{n} P_{n}=\alpha_{n}\left(\cdot, e_{n}\right) e_{n}, L_{n}=L_{0}-\mathrm{i} V_{n}=$ $-\mathrm{i} V_{n}$ and combining (8.5) with (8.6), we obtain

$$
\Delta_{L_{1} / L_{0}}(z)=\prod_{n \geq 0}\left(1-\mathrm{i} \alpha_{n} z^{-1}\right)=\lim _{N \rightarrow \infty} \prod_{n=0}^{N}\left(1-\mathrm{i} \alpha_{n} z^{-1}\right) .
$$

By Lemma 5.5, (i) of [MN2], there is a non-negative function $\eta_{n}$ in $L^{1}(\mathbb{R})$ such that

$$
\begin{aligned}
u_{n}(z) & \stackrel{\text { def }}{=} \Delta_{L_{n} / L_{n-1}}(z) \stackrel{\text { def }}{=} \operatorname{det}\left(I+\mathrm{i} V_{n}\left(L_{n-1}-z I\right)^{-1}\right) \\
& =1-\mathrm{i} \alpha_{n} z^{-1}=\exp \left(\mathrm{i} \pi^{-1} \int_{\mathbb{R}} \eta_{n}(t)(t-z)^{-1} d t\right), \quad z \in \mathbb{C}_{-},
\end{aligned}
$$

where

$$
\eta_{n}(t)=\lim _{y \uparrow 0} \log \left(\left|u_{n}(t-i y)\right|\right)=\log \left(\left|1-\mathrm{i} \alpha_{n} t^{-1}\right|\right)=(1 / 2) \log \left(1+\alpha_{n}^{2} t^{-2}\right) \geq 0 .
$$

Integrating by parts, we see that $\eta_{n} \in L^{1}(\mathbb{R})$ and

$$
\sum_{n \geq 0} \int_{\mathbb{R}} \eta_{n}(t)=\sum_{n \geq 0} \int_{0}^{\infty} \log \left(1+\frac{\alpha_{n}^{2}}{t^{2}}\right) d t=2 \sum_{n \geq 0} \int_{0}^{\infty} \frac{\alpha_{n}^{2}}{t^{2}+\alpha_{n}^{2}} d t=\pi \sum_{n \geq 0} \alpha_{n}=\pi C_{0} .
$$

By the B. Levi theorem, the function $\eta$,

$$
\eta(t) \stackrel{\text { def }}{=} \sum_{n \geq 0} \eta_{n}(t)=(1 / 2) \sum_{n \geq 0} \log \left(1+\alpha_{n}^{2} t^{-2}\right),
$$

is well defined for almost all $t \in \mathbb{R}$, non-negative, and $\eta \in L^{1}(\mathbb{R})$. Combining relations (8.7)-(8.10), we arrive at the representation

$$
\Delta_{L_{1} / L_{0}}(z)=\exp \left(\mathrm{i} \pi^{-1} \int_{\mathbb{R}} \eta(x)(x-z)^{-1} d x\right) \quad z \in \mathbb{C}_{-} .
$$

Taking the logarithmic derivative one arrives at trace formula (7.1) showing that i $\eta$ is a (purely imaginary) spectral shift functions for the pair $\left\{L_{1}, L_{0}\right\}$, i.e., i $\eta \in \mathscr{S}\left\{L_{1}, L_{0}\right\}$.

(ii) It follows from (8.9) that the harmonic conjugate to $\eta_{n}$ function $\widetilde{\eta}_{n}$ is given by $\widetilde{\eta}_{n}(t)=\arctan \left(\alpha_{n} / t\right), t \in \mathbb{R}, n \geq 0$, and

$$
\widetilde{\eta}(t)=-\lim _{y \uparrow 0} \frac{1}{\pi} \int_{\mathbb{R}} \frac{\eta(x)(x-t)}{(x-t)^{2}+y^{2}} d x=\sum_{j \geq 0} \widetilde{\eta}_{j}(t)=\sum_{j \geq 0} \arctan \frac{\alpha_{n}}{t}, \quad t \in \mathbb{R},
$$

where the convergence is pointwise. Clearly, $\widetilde{\eta}$ is real-valued and $\widetilde{\eta}+\mathrm{i} \eta \in H^{1}\left(\mathbb{R},\left(1+t^{2}\right)^{-1}\right)$ if and only if $\widetilde{\eta} \in L^{1}\left(\mathbb{R},\left(1+t^{2}\right)^{-1}\right)$. Therefore, by Proposition 3.8 of [MN2], a real spectral 
shift function for $\left\{L_{1}, L_{0}\right\}$ exists if and only if $\widetilde{\eta} \in L^{1}\left(\mathbb{R},\left(1+t^{2}\right)^{-1}\right)$. Thus, it remains to show that the latter inclusion is equivalent to condition (8.4).

Making use the change of variables $x=\alpha_{j} / t$ we obtain

$$
\begin{aligned}
& \int_{\mathbb{R}} \frac{|\widetilde{\eta}(t)|}{1+t^{2}} d t=\sum_{j=1}^{\infty} \int_{\mathbb{R}} \frac{\left|\widetilde{\eta}_{j}(t)\right|}{1+t^{2}} d t=2 \sum_{j=1}^{\infty} \int_{0}^{\infty} \frac{\widetilde{\eta}_{j}(t)}{1+t^{2}} d t=2 \sum_{j=1}^{\infty} \int_{0}^{\infty} \frac{\arctan \left(\alpha_{j} / t\right)}{1+t^{2}} d t \\
& =2 \sum_{j=1}^{\infty} \alpha_{j} \int_{0}^{\infty} \frac{\arctan x}{\alpha_{j}^{2}+x^{2}} d x=2 \sum_{j=1}^{\infty} \alpha_{j} \int_{0}^{1} \frac{\arctan x}{\alpha_{j}^{2}+x^{2}} d x+2 \sum_{j=1}^{\infty} \alpha_{j} \int_{1}^{\infty} \frac{\arctan x}{\alpha_{j}^{2}+x^{2}} d x .
\end{aligned}
$$

It is easily seen that for some $c_{0}>0$,

$$
c_{0} x \leq \arctan x \leq x, \quad x \in[0,1], \quad \text { and } \quad \int_{1}^{\infty}(\arctan x)\left(\alpha_{j}^{2}+x^{2}\right)^{-1} d x \leq \frac{\pi}{2} .
$$

Combining these estimates with (8.12) leads to two-sided estimate

$$
c_{0} \sum_{j} \alpha_{j} \int_{0}^{1} \frac{2 x}{x^{2}+\alpha_{j}^{2}} d x \leq \int_{\mathbb{R}} \frac{|\widetilde{\eta}(t)|}{1+t^{2}} d t \leq \sum_{j} \alpha_{j} \int_{0}^{1} \frac{2 x}{x^{2}+\alpha_{j}^{2}} d x+\pi C_{1},
$$

where $C_{1}=\sum_{j \geq 0} \alpha_{j}$. To complete the proof, it remains to observe that

$$
\int_{0}^{1} 2 x\left(\alpha_{j}^{2}+x^{2}\right)^{-1} d x=\log \left(\left(1+\alpha_{j}^{2}\right) \alpha_{j}^{-2}\right) \sim-2 \log \alpha_{j}=2\left|\log \alpha_{j}\right| \quad \text { as } \quad j \rightarrow \infty .
$$

Using the Cayley transform, we deduce the following result.

Corollary 8.4. Let $T_{0}=I$ and let $T_{1}$ be a self-adjoint contraction such that $I-T_{1} \in$ $\boldsymbol{S}_{1}$ and let $\left\{\lambda_{n}\right\}_{n \geq 0}$ be the sequence of eigenvalues of $T_{1}$ counted with multiplicities. If $\operatorname{Ker}(I \pm T)=\{\mathbf{0}\}$, then $\mathscr{S}\left\{T_{1}, T_{0}\right\}$ contains no real function if and only if

$$
\sum_{n}\left(1-\lambda_{n}\right)\left(1+\lambda_{n}\right)^{-1}\left|\log \left(\left(1-\lambda_{n}\right)\left(1+\lambda_{n}\right)^{-1}\right)\right|=\infty
$$

Proof. Let $L_{0}$ and $L_{1}$ be the Cayley transforms of $T_{0}$ and $T_{1}$ (see (7.3)). Clearly, $L_{0}=\mathbf{0}$ and $L_{1}=-\mathrm{i} V$, where $V=\left(I-T_{1}\right)\left(I+T_{1}\right)^{-1} \in \boldsymbol{S}_{1}$ and $V \geq 0$. Therefore condition (8.4) is transformed into condition (8.14). It remains to apply Theorem 8.3 and take formula (7.5) into account.

Remark. In connection with Theorem 7.2 and Corollary 8.4 we mention Theorem 3.3 from $[\mathrm{AN}]$. It says that the class $\mathscr{S}\left\{T_{1}, T_{0}\right\}$ contains a real function whenever in addition to the condition $T_{1}-T_{0} \in \boldsymbol{S}_{1}$ the following conditions are satisfied: $I-\left|T_{1}\right| \in \boldsymbol{S}_{1}^{0}$ and $I-\left|T_{0}^{*}\right| \in \boldsymbol{S}_{1}^{0}$, where $\boldsymbol{S}_{1}^{0} \stackrel{\text { def }}{=}\left\{A: \sum_{j} s_{j}(A)\left|\log s_{j}(A)\right|<\infty\right\} \subset \boldsymbol{S}_{1}$ and $\left\{s_{j}(A)\right\}_{j \geq 0}$ is the sequence of singular values of an operator $A$. Theorem 8.3 shows that Theorem 3.3 of $[\mathrm{AN}]$ is sharp.

In [MSZ] the authors studied spectral shift functions for a pair $\left\{L_{0}, L_{0}-\mathrm{i} V\right\}$ with $L_{0}=$ $L_{0}^{*}$ and $0 \leq V \in \boldsymbol{S}_{1}$. In particular, it was shown that $w_{2}(t)=\lim _{y \downarrow 0} \arg \left(\operatorname{det}_{L_{1} / L_{0}}(t+\mathrm{i} y)\right)$ is in $L^{1}(\mathbb{R})$ if and only if the operator $L_{0}-\mathrm{i} V$ is self-adjoint. 


\section{The main results}

In this section we establish the main results of the paper. The following theorem

Theorem 9.1. Let $T_{1}$ and $T_{0}$ be contractions such that $T_{1}-T_{0} \in \boldsymbol{S}_{1}$ and let $f \in \mathrm{OL}_{\mathrm{A}}$. Then $f\left(T_{1}\right)-f\left(T_{0}\right) \in \boldsymbol{S}_{1}$ and the following trace formula holds

$$
\operatorname{trace}\left(f\left(T_{1}\right)-f\left(T_{0}\right)\right)=\int_{\mathbb{T}} f^{\prime}(\zeta) \boldsymbol{\xi}(\zeta) d \zeta
$$

for an arbitrary spectral shift function $\boldsymbol{\xi}$ for the pair $\left\{T_{1}, T_{0}\right\}$. In particular,

$$
\int_{\mathbb{T}} \boldsymbol{\xi}(\zeta) d \zeta=\operatorname{trace}\left(T_{1}-T_{0}\right)
$$

Conversely, if for a function $f$ in $\mathrm{C}_{\mathrm{A}}, f\left(T_{1}\right)-f\left(T_{0}\right) \in \boldsymbol{S}_{1}$ whenever $T_{1}$ and $T_{0}$ are contractions such that $T_{1}-T_{0} \in \boldsymbol{S}_{1}$, then $f \in \mathrm{OL}_{\mathrm{A}}$.

Proof. By Theorem 6.1, formula (6.1) holds for some complex Borel measure $\nu$. On the other hands, by Theorem 7.2, if $\boldsymbol{\xi} \in \mathscr{S}\left\{T_{1}, T_{0}\right\}$, then formula (9.1) in the case $f(z)=(z-\lambda)^{-1},|\lambda|>1$. Since the linear combinations of such functions are dense in

the class $X \stackrel{\text { def }}{=}\left\{f: f^{\prime} \in H^{1}\right\}$, it follows that $\int_{\mathbb{T}} f^{\prime}(\zeta) \xi(\zeta) d \zeta=\int_{\mathbb{T}} f^{\prime}(\zeta) d \nu(\zeta)$ for every $f$ in $X$. This implies the following equality for the Fourier coefficients: $\widehat{\boldsymbol{\xi}}(j)=\widehat{\nu}(j), j<0$. By the brothers Riesz theorem (see [Ko], Ch. II, Sect. A), $\nu$ is absolutely continuous with respect to Lebesgue measure and its Radon-Nikodym density is a spectral shift functions for the pair $\left\{T_{1}, T_{0}\right\}$. This implies formula (9.1) for an arbitrary function $f$ in $\mathrm{OL}_{\mathrm{A}}$. To get formula (9.2), it suffices to put $f(z)=z$.

The last conclusion of the theorem is an immediate consequence of the corresponding fact for functions of unitary operators, see [AP2] and the fact that $\mathrm{OL}_{\mathrm{A}}=\mathrm{OL}_{\mathbb{T}} \cap C_{\mathrm{A}}$ (see $[\mathrm{KS}]$ and $[\mathrm{AP} 1])$.

Theorem 9.2. Let $\left\{L_{1}, L_{0}\right\}$ be a pair of resolvent comparable maximal dissipative operators and let $f$ be a function analytic in $\mathbb{C}_{+}$and such that the function

$$
\zeta \mapsto f\left((\mathrm{i}-\zeta)(\mathrm{i}+\zeta)^{-1}\right), \quad \zeta \in \mathbb{C}_{+},
$$

belongs to $\mathrm{OL}_{\mathrm{A}}$. Then $f\left(L_{1}\right)-f\left(L_{0}\right) \in \boldsymbol{S}_{1}$ and

$$
\operatorname{trace}\left(f\left(L_{1}\right)-f\left(L_{0}\right)\right)=\int_{\mathbb{R}} f^{\prime}(t) \boldsymbol{\omega}(t) d t,
$$

where $\boldsymbol{\omega}$ is a spectral shift function for the pair $\left\{L_{1}, L_{0}\right\}$.

Proof. The theorem can be proved by passing to the contractions $T_{0}=\left(\mathrm{i} I-L_{0}\right)(\mathrm{i} I+$ $\left.L_{0}\right)^{-1}$ and $T_{1}=\left(\mathrm{i} I-L_{1}\right)\left(\mathrm{i} I+L_{1}\right)^{-1}$ and applying Theorem 9.1.

Note that Theorem 9.2 eliminates the additional assumption $\left(\rho\left(L_{0}\right) \cup \sigma_{\mathrm{c}}\left(L_{0}\right)\right) \cap \mathbb{C}_{+} \neq \varnothing$ in the statement of Theorem 7.1. 


\section{ApPEndix A. Pairs of accumulative and dissipative operators}

A1. Auxiliary results. Here we improve Theorem 3.13 from [MN2] mentioned in the Introduction. To this end we need several auxiliary results.

Recall (see $[\mathrm{SNF}]$ ) that a bounded holomorphic function $W: \mathbb{C}_{+} \rightarrow \mathcal{B}(\mathscr{H})$ is called an outer function if multiplication by $W$ on $H_{\mathscr{H}}^{2}\left(\mathbb{C}_{+}\right)$has dense range. Recall that the strong limit $W(t) \stackrel{\text { def }}{=} \lim _{y \downarrow 0} W(t+\mathrm{i} y)$ exists for almost all $t \in \mathbb{R}$, see [SNF].

Lemma A.1. Let $W: \mathbb{C}_{+} \rightarrow \mathcal{B}(\mathscr{H})$ be a contractive holomorphic function. If $\operatorname{Re} W(z)>\mathbf{0}$ for $z \in \mathbb{C}_{+}$and $\operatorname{Ker} \operatorname{Re} W(t)=\{\mathbf{0}\}$ a.e. on $\mathbb{R}$, then $W$ is an outer function.

Proof. Assume the contrary. Then there exists $g \in H_{\mathscr{H}}^{2}\left(\mathbb{C}_{+}\right)$such that

$$
(W f, g)_{H_{\mathscr{H}}^{2}}=\int_{-\infty}^{\infty}(W(t) f(t), g(t))_{\mathscr{H}} d t=0, \quad \text { for all } \quad f \in H_{\mathscr{H}}^{2}\left(\mathbb{C}_{+}\right) .
$$

Putting $f=g$ in (A.1), taking the real part, and observing that $\operatorname{Re} \Theta(t) \geq 0$ for a.e. $t \in \mathbb{R}$, yields $\int_{\mathbb{R}}\left\|(\operatorname{Re} W(t))^{1 / 2} g(t)\right\|_{\mathscr{H}}^{2} d t=0$, and so $(\operatorname{Re} W(t)) g(t)=0$ a.e. on $\mathbb{R}$. Hence, $g(t)=0$ a.e. and multiplication by $W$ has dense range in $H_{\mathscr{H}}^{2}\left(\mathbb{C}_{+}\right)$.

Definition. A holomorphic function $F: \mathbb{C}_{+} \cup \mathbb{C}_{-} \rightarrow \mathcal{B}(\mathscr{H})$ is called an $R$-function (in short $F \in R[\mathscr{H}]$ ) if $\operatorname{Im} z \cdot \operatorname{Im} F(z)>0$ and $F(z)^{*}=F(\bar{z})$ for $z \in \mathbb{C}_{ \pm}$. We also use the notation $R^{\mathrm{u}}[\mathscr{H}]$ for $\left\{F \in R[\mathcal{H}]: 0 \in \rho(\operatorname{Im} F(z)), z \in \mathbb{C}_{ \pm}\right\}$.

Lemma A.2. Let $M \in R^{\mathrm{u}}[\mathscr{H}]$ and $B$ a bounded accumulative operator on $\mathscr{H}$. Then (i) If $0 \leq V_{+} \leq|\operatorname{Im} B|=-\operatorname{Im} B \geq 0$ and $V_{+} \in \boldsymbol{S}_{1}$, then the function $w_{+}$ $\stackrel{\text { def }}{=} \operatorname{det}\left(I+\mathrm{i} V_{+}(B-M)^{-1}\right)$ is holomorphic and contractive in $\mathbb{C}_{+}$. Moreover, $w_{+}$is an outer function, and so it admits a representation

$$
w_{+}(z)=\varkappa_{+} \exp \left(\frac{\mathrm{i}}{\pi} \int_{\mathbb{R}}\left((t-z)^{-1}-t\left(1+t^{2}\right)^{-1}\right) \eta_{+}(t) d t\right), \quad z \in \mathbb{C}_{+},
$$

where $\eta_{+}(t)=-\log \left|w_{+}(t)\right| \geq 0$ a.e. on $\mathbb{R}, \eta_{+} \in L^{1}\left(\mathbb{R},\left(1+t^{2}\right)^{-1}\right)$, and $\varkappa_{+} \in \mathbb{T}$.

(ii) If $V \leq|\operatorname{Im} B|=-\operatorname{Im} B$ and $V=V^{*} \in \boldsymbol{S}_{1}$, then $w \stackrel{\text { def }}{=} \operatorname{det}\left(I+\mathrm{i} V(B-M)^{-1}\right)$ is an outer function in $\mathbb{C}_{+}$and admits a representation

$$
w(z)=\varkappa \exp \left(\frac{\mathrm{i}}{\pi} \int_{\mathbb{R}}\left((t-z)^{-1}-t\left(1+t^{2}\right)^{-1}\right) \eta(t) d t\right), \quad z \in \mathbb{C}_{+}, \quad \varkappa_{+} \in \mathbb{T},
$$

with a real function $\eta \in L^{1}\left(\mathbb{R},\left(1+t^{2}\right)^{-1}\right)$. Besides, $\eta(t)=-\log |w(t)|$ a.e. on $\mathbb{R}$.

Proof. (i) Put

$$
W_{+}(z) \stackrel{\text { def }}{=} I_{\mathscr{H}}+\mathrm{i} V_{+}{ }^{1 / 2}(B-M(z))^{-1} V_{+}{ }^{1 / 2}, \quad z \in \mathbb{C}_{+} .
$$

First, we show that $W_{+}$is a holomorphic contractive $\mathscr{H}$-valued function in $\mathbb{C}_{+}$. Since $\operatorname{Im} M(z) \geq \varepsilon(z) I, z \in \mathbb{C}_{+}$, for a positive function $\varepsilon$ on $\mathbb{C}_{+}$and $\operatorname{Im} B \leq \mathbf{0}$, the function $(B-M)^{-1}$ is well defined and holomorphic in $\mathbb{C}_{+}$. Besides, taking into account the inequality $\left(\operatorname{Im} B+V_{+}\right) \leq \mathbf{0}$, we get

$I-W_{+}^{*}(z) W_{+}(z)=V_{+}^{1 / 2}\left(B^{*}-M^{*}(z)\right)^{-1}\left(2 \operatorname{Im} M(z)-2 \operatorname{Im} B-V_{+}\right)(B-M(z))^{-1} V_{+}^{1 / 2} \geq \mathbf{0}$. 
Hence, $W_{+}$is contractive. Next, we show that $W_{+}$is an outer $\mathcal{B}(\mathscr{H})$-valued function in $\mathbb{C}_{+}$. To this end we set $C \stackrel{\text { def }}{=} B+\mathrm{i} V_{+}$and

$$
W_{-}(z) \stackrel{\text { def }}{=} W_{+}(z)^{-1}=I_{\mathcal{H}}-\mathrm{i} V_{+}^{1 / 2}(C-M(z))^{-1} V_{+}^{1 / 2}, \quad z \in \mathbb{C}_{+} .
$$

Besides, keeping in mind that $\operatorname{Im} C=\operatorname{Im} B+V_{+} \leq 0$, one can easily obtain

$$
\operatorname{Re} W_{-}(z)=I_{\mathscr{H}}+V_{+}^{1 / 2}(C-M(z))^{-1}\left(-\operatorname{Im} C+\operatorname{Im} M(z)\left(C^{*}-M^{*}(z)\right)^{-1} V_{+}^{1 / 2} \geq I_{\mathcal{H}}\right. \text { (A.6) }
$$

for $z \in \mathbb{C}_{+}$. It follows with account of (A.5) that

$$
\operatorname{Re} W_{+}(z)=W_{-}(z)^{-1}\left(\operatorname{Re} W_{-}(z)\right)\left(W_{-}(z)^{-1}\right)^{*} \geq W_{-}(z)^{-1}\left(W_{-}(z)^{-1}\right)^{*}>\mathbf{0} .
$$

Since $V \in \boldsymbol{S}_{1}$, the limits $W_{ \pm}(x)=\lim _{y \downarrow 0} W_{ \pm}(x+\mathrm{i} y)$ exist in the $\boldsymbol{S}_{2}$-norm for a.e. $x \in \mathbb{R}$. Therefore passing to the limit in the identity $W_{ \pm}(x+\mathrm{i} y) W_{\mp}(x+\mathrm{i} y)=I$ as $y \downarrow 0$, we obtain $W_{ \pm}(x) W_{\mp}(x)=I$ a.e. on $\mathbb{R}$. Passing to the limit as $y \downarrow 0$ in (A.7) we obtain

$$
\operatorname{Re} W_{+}(x)=W_{-}(x)^{-1}\left(\operatorname{Re} W_{-}(x)\right)\left(W_{-}(x)^{-1}\right)^{*} \geq W_{-}(x)^{-1}\left(W_{-}(x)^{-1}\right)^{*}>\mathbf{0} \quad \text { a.e.. }
$$

Hence, Ker Re $W_{+}(x)=\{0\}$ a.e. on $\mathbb{R}$. By Lemma A.1, $W_{+}$is an outer function in $\mathbb{C}_{+}$and so is $w_{+}(z)=\operatorname{det} W_{+}(z)$ (see $[\mathrm{SNF}]$ ). Being contractive and outer in $\mathbb{C}_{+}$, the function $w_{+}$admits a representation (A.2) (see [Ko], Sect. VI C).

(ii) Let $V=V_{+}-V_{-}, V_{ \pm} \geq 0$. Put $B_{-} \stackrel{\text { def }}{=} B-\mathrm{i} V_{-}$. Since $\operatorname{Im} B_{-}=\operatorname{Im} B-V_{-} \leq \mathbf{0}$, the operator $B_{-}$is accumulative. One can easily check that for $z \in \mathbb{C}_{+}$,

$\operatorname{det}\left(I+\mathrm{i} V(B-M(z))^{-1}\right)=\operatorname{det}\left(I+\mathrm{i} V_{+}\left(B_{-}-M(z)\right)^{-1}\right)\left(\operatorname{det}\left(I+\mathrm{i} V_{-}\left(B_{-}-M(z)\right)^{-1}\right)\right)^{-1}$.

The assumption $V \leq-\operatorname{Im} B$ yields $0 \leq V_{+} \leq-\operatorname{Im} B+V_{-}=-\operatorname{Im} B_{-}$. In accordance with (i) there exist a non-negative $\eta_{+} \in L^{1}\left(\mathbb{R},\left(1+t^{2}\right)^{-1}\right)$ and $\varkappa_{+} \in \mathbb{T}$ such that

$\operatorname{det}\left(I+\mathrm{i} V_{+}\left(B_{-}-M(z)\right)^{-1}\right)=\varkappa_{+} \exp \left(\frac{\mathrm{i}}{\pi} \int_{\mathbb{R}}\left((t-z)^{-1}-t\left(1+t^{2}\right)^{-1}\right) \eta_{+}(t) d t\right), \quad z \in \mathbb{C}_{+}$.

Similarly, since $\mathbf{0} \leq V_{-} \leq-\operatorname{Im} B+V_{-}=-\operatorname{Im} B_{-}$and (i) ensures a representation

$\operatorname{det}\left(I+\mathrm{i} V_{-}\left(B_{-}-M(z)\right)^{-1}\right)=\varkappa_{-} \exp \left(\frac{\mathrm{i}}{\pi} \int_{\mathbb{R}}\left((t-z)^{-1}-t\left(1+t^{2}\right)^{-1}\right) \eta_{-}(t) d t\right), z \in \mathbb{C}_{+}$

for a nonnegative function $\eta_{-} \in L^{1}\left(\mathbb{R},\left(1+t^{2}\right)^{-1}\right)$ and $\varkappa_{-} \in \mathbb{T}$. Setting $\varkappa_{\text {def }}^{=} \varkappa_{+} / \varkappa_{-} \in \mathbb{T}$ and $\eta \stackrel{\text { def }}{=} \eta_{+}-\eta_{-}$, we arrive at (A.3).

The following lemma generalizes Krein's result [Kr1] on exponential representation of the perturbation determinant of a pair of selfadjoint operators.

Lemma A.3. Let $M \in R^{\mathrm{u}}[\mathscr{H}]$, let $B$ a bounded accumulative (possibly, self-adjoint) operator on $\mathscr{H}$ and let $V=V^{*} \in \boldsymbol{S}_{1}$. Then there exist a real-valued function $\xi$ in $L^{1}\left(\mathbb{R},\left(1+t^{2}\right)^{-1}\right)$ and a constant $c>0$ such that the equality

$$
\operatorname{det}\left(I+V(B-M(z))^{-1}\right)=c \exp \left(\frac{1}{\pi} \int_{\mathbb{R}}\left((t-z)^{-1}-t\left(1+t^{2}\right)^{-1}\right) \xi(t) d t\right)
$$

holds for $z \in \mathbb{C}_{+}$. Moreover, if $V=V_{+} \geq 0$, then $\xi=\xi_{+} \geq 0$. 
Proof. (i) Assume first that $V=V_{+} \geq \mathbf{0}$. Consider the operator-valued Nevanlinna function $\Omega_{+}, \Omega_{+}(z) \stackrel{\text { def }}{=} I+V_{+}{ }^{1 / 2}(B-M(z))^{-1} V_{+}{ }^{1 / 2}, z \in \mathbb{C}_{+}$. Since $\Omega_{+}(z)$ is $m$-dissipative for $z \in \mathbb{C}_{+}$and $0 \in \rho\left(\Omega_{+}(z)\right), z \in \mathbb{C}_{+}$, the operator-valued function $\log \left(\Omega_{+}(z)\right)$ is welldefined for $z \in \mathbb{C}_{+}$(see $\left.[\mathrm{GMN}]\right)$. Besides, since $V_{+} \in \boldsymbol{S}_{1}$, we have $\log \left(\Omega_{+}(z)\right) \in \boldsymbol{S}_{1}$ and Theorem 2.8 of [GMN]) ensures the existence of a non-negative measurable function $\Xi_{+}: \mathbb{R} \rightarrow \boldsymbol{S}_{1}$ such that the following representation holds

$$
\log \left(\Omega_{+}(z)\right)=\Omega_{+}+\frac{1}{\pi} \int_{\mathbb{R}}\left(\frac{1}{t-z}-\frac{t}{1+t^{2}}\right) \Xi_{+}(t) d t, \quad z \in \mathbb{C}_{+}, \quad \int_{\mathbb{R}} \frac{\Xi_{+}(t)}{1+t^{2}} d t \in \mathcal{B}(\mathscr{H}) .
$$

Here the integral is understood in the weak sense and $\Omega_{+}=\Omega_{+}^{*} \in \boldsymbol{S}_{1}$. Taking the traces in this identity and setting $\xi_{+}(t) \stackrel{\text { def }}{=} \operatorname{trace}\left(\Xi_{+}(t)\right), t \in \mathbb{R}$, we arrive at the representation

$$
\operatorname{trace}\left(\log \left(\Omega_{+}(z)\right)\right)=\operatorname{trace}\left(\Omega_{+}\right)+\frac{1}{\pi} \int_{\mathbb{R}}\left((t-z)^{-1}-t\left(1+t^{2}\right)^{-1}\right) \xi_{+}(t) d t, \quad z \in \mathbb{C}_{+} .
$$

Taking into account the identity $\operatorname{det}(I+G)=e^{\operatorname{trace}(\log (I+G))}$ valid for any dissipative $G \in \mathfrak{S}_{1}(\mathfrak{H})$, we arrive at (A.8) with $V=V_{+}, \xi=\xi_{+}$, and $c=c_{+}=\exp \left\{\operatorname{tr}\left(\Omega_{+}\right)\right\}>0$.

(ii) Passing to the general case, we start with the spectral decomposition $V=V_{+}-V_{-}$ $\left(V_{ \pm} \geq \mathbf{0}, V_{ \pm} V_{\mp}=\mathbf{0}\right)$, and set $B_{-} \stackrel{\text { def }}{=} B-V_{-}$. It follows from the identity

$$
\left(I+V(B-M(z))^{-1}\right)\left(I+V_{-}\left(B_{-}-M(z)\right)^{-1}\right)=I+V_{+}\left(B_{-}-M(z)\right)^{-1}
$$

that for

$$
\operatorname{det}\left(I+V(B-M(z))^{-1}\right)=\frac{\operatorname{det}\left(I+V_{+}\left(B_{-}-M(z)\right)^{-1}\right)}{\operatorname{det}\left(I+V_{-}\left(B_{-}-M(z)\right)^{-1}\right)}, \quad z \in \mathbb{C}_{ \pm} .
$$

Note that representations (A.8) with $V_{ \pm} \geq \mathbf{0}$ and $\xi_{ \pm} \geq 0$ instead of $V$ and $\xi$ have just been established in (i). Combining these representations with (A.9) and setting $\xi \stackrel{\text { def }}{=} \xi_{+}-\xi_{-}$, we arrive at (A.8).

A2. Boundary triplets and Weyl functions. Let us recall basic facts of the theory of boundary triplets. Let $A$ be a densely defined symmetric operator in $\mathscr{H}$.

Definition. A triplet $\Pi=\left\{\mathscr{K}, \Gamma_{0}, \Gamma_{1}\right\}$, where $\mathscr{K}$ is an auxiliary Hilbert space and $\Gamma_{0}, \Gamma_{1}: \operatorname{dom}\left(A^{*}\right) \rightarrow \mathscr{K}$ are linear operators, is called a boundary triplet for $A^{*}$ if "Green's identity"

$$
\left(A^{*} f, g\right)-\left(f, A^{*} g\right)=\left(\Gamma_{1} f, \Gamma_{0} g\right)_{\mathscr{K}}-\left(\Gamma_{0} f, \Gamma_{1} g\right)_{\mathscr{K}}, \quad f, g \in \operatorname{dom}\left(A^{*}\right),
$$

holds and the mapping $\Gamma=\left(\Gamma_{0}, \Gamma_{1}\right): \operatorname{dom}\left(A^{*}\right) \rightarrow \mathcal{H} \oplus \mathcal{H}$ is surjective.

A boundary triplet $\Pi=\left\{\mathscr{K}, \Gamma_{0}, \Gamma_{1}\right\}$ for $A^{*}$ exists whenever $A$ has equal deficiency indices $n_{+}(A)=n_{-}(A)$. Note also that $n_{ \pm}(A)=\operatorname{dim}(\mathscr{K})$. With any boundary triplet $\Pi$ we can associate a self-adjoint extension $A_{0}$ of $A, A_{0} \stackrel{\text { def }}{=} A^{*} \mid \operatorname{ker}\left(\Gamma_{0}\right)=A_{0}^{*}$.

A closed extension $\widetilde{A}$ of $A$ is called proper if $A \subseteq \widetilde{A} \subseteq A^{*}$. The set of proper extensions is denoted by $\operatorname{Ext}_{A}$. A dissipative (accumulative) extension of $A$ is always proper.

The role of a boundary triplet in extension theory is similar to the role of a coordinate system in analytic geometry. In particular, it allows one to parameterize the set Ext $A$ by means of the set $\widetilde{\mathcal{C}}(\mathcal{H})$ of closed linear relations in $\mathscr{K}$ (i.e., subspaces in $\mathscr{K} \oplus \mathscr{K}$ ). 
Lemma A.4 ([DM]). Let $\Pi=\left\{\mathscr{K}, \Gamma_{0}, \Gamma_{1}\right\}$ be a boundary triplet for $A^{*}$. The map

$$
\operatorname{Ext}_{A} \ni \widetilde{A} \rightarrow \Theta \stackrel{\operatorname{def}}{=} \operatorname{\Gamma dom}(\widetilde{A})=\left\{\left\{\Gamma_{0} f, \Gamma_{1} f\right\}: f \in \operatorname{dom}(\widetilde{A})\right\} \in \widetilde{\mathcal{C}}(\mathscr{K})
$$

establishes a bijective correspondence between $\operatorname{Ext}_{A}$ and the set of all linear relations in $\mathscr{K}$. We write $\widetilde{A}=A_{\Theta}$ if $\widetilde{A}$ corresponds to $\Theta$ via (A.11). Moreover, the following holds:

(i) $A_{\Theta}$ is symmetric (self-adjoint) if and only if $\Theta$ is symmetric (self-adjoint).

(ii) $A_{\Theta}$ is $m$-dissipative ( $m$-accumulative) if and only if so is $\Theta$.

(iii) The extensions $A_{\Theta}$ and $A_{0}$ are disjoint, i.e., $\operatorname{dom} A_{W} \cap \operatorname{dom} A_{0}=\operatorname{dom} A$, if and only if $\Theta=\operatorname{graph}(B)$ and $B$ is a closed operator in $\mathscr{K}$, and (A.11) takes the form

$$
\widetilde{A}=A_{B} \stackrel{\text { def }}{=} A_{\text {graph }(B)}=A^{*} \mid \operatorname{ker}\left(\Gamma_{1}-B \Gamma_{0}\right) .
$$

The operator $B$ is called the boundary operator of $\widetilde{A}$ with respect to the triplet $\Pi$.

Definition ([MN1]). A boundary triplet $\Pi=\left\{\mathscr{K}, \Gamma_{0}, \Gamma_{1}\right\}$ for $A^{*}$ is called regular for the family $\left\{\widetilde{A}_{j}\right\}_{j=1}^{N}$ of proper extensions if there exist bounded operators $B_{j} \in \mathcal{B}(\mathscr{K})$ such that $\widetilde{A}_{j}=A_{B_{j}} \stackrel{\text { def }}{=} A^{*} \mid \operatorname{Ker}\left(\Gamma_{1}-B_{j} \Gamma_{0}\right), j \in\{1, \ldots, N\}$, (cf. (A.12)).

Theorem A.5. ([MN1], Th. 3.5). Let $A$ be as above and let $\left\{\widetilde{A}_{j}\right\}_{j=1}^{N} \subset \operatorname{Ext}_{A}$ and $\widetilde{A}_{N+1}=\widetilde{A} \in \operatorname{Ext}_{A}$. Assume also that $\bigcap_{j=1}^{N+1} \rho\left(\widetilde{A}_{j}\right) \neq \varnothing$ and

$$
\left(\widetilde{A}-z_{1} I\right)^{-1}-\left(\widetilde{A}_{j}-z_{1} I\right)^{-1} \in \boldsymbol{S}_{\infty}, \quad z_{1} \in \bigcap_{k=1}^{N+1} \rho\left(\widetilde{A}_{k}\right), \quad j \in\{1, \ldots, N\} .
$$

If there is $z_{2} \in \rho(\widetilde{A}) \cup \sigma_{\mathrm{c}}(\widetilde{A})$ such that $\operatorname{Im}\left(z_{1}\right) \operatorname{Im}\left(z_{2}\right)<0$, then the family $\left\{\widetilde{A}_{j}\right\}_{j=1}^{N+1}$ admits a regular boundary triplet. In particular, the last condition is satisfied if $\rho(\widetilde{A}) \cap \mathbb{C}_{ \pm} \neq \varnothing$.

The main analytical tool in this theory is played by the so-called Weyl function.

Definition $([\mathrm{DM}])$. Let $\Pi=\left\{\mathscr{K}, \Gamma_{0}, \Gamma_{1}\right\}$ be a boundary triplet for $A^{*}, A_{0}=$ $A^{*} \mid \operatorname{ker}\left(\Gamma_{0}\right)$, and $\mathfrak{N}_{z} \stackrel{\text { def }}{=} \operatorname{ker}\left(A^{*}-z I\right)$. The operator valued functions $\gamma: \rho\left(A_{0}\right) \rightarrow$ $\mathcal{B}(\mathscr{K}, \mathscr{H})$ and $M: \rho\left(A_{0}\right) \rightarrow \mathcal{B}(\mathcal{H})$ defined by

$$
\gamma(z)=\left(\Gamma_{0} \mid \mathfrak{N}_{z}\right)^{-1} \quad \text { and } \quad M(z)=\Gamma_{1} \gamma(z), \quad z \in \rho\left(A_{0}\right),
$$

are called the $\gamma$-field and Weyl function corresponding to $\Pi$.

An important property of the Weyl function is that $z \in \rho\left(A_{\Theta}\right) \cap \rho\left(A_{0}\right)$ if and only if $0 \in \rho(\Theta-M(z))$. Note that $M \in R^{\mathrm{u}}[\mathscr{K}]$ and the following Krein-type formula holds:

$$
\left(A_{\Theta}-z I\right)^{-1}-\left(A_{0}-z I\right)^{-1}=\gamma(z)(\Theta-M(z))^{-1} \gamma(\bar{z})^{*}, \quad z \in \rho\left(A_{0}\right) \cap \rho\left(A_{\Theta}\right) .
$$

A3. Perturbation determinants. Next, we recall some basic facts on perturbation determinants treated in the framework of boundary triplets (see [MN1] for details). If $\widetilde{A}^{\prime}, \widetilde{A} \in \operatorname{Ext}_{A}$ and meet the assumptions of Theorem A.5, then there is a regular boundary triplet for $\left\{\widetilde{A}^{\prime}, \widetilde{A}\right\}$, i.e., $\widetilde{A}^{\prime}=A_{B^{\prime}}, \widetilde{A}=A_{B}$, with $B^{\prime}, B \in \mathcal{B}(\mathscr{K})$ and by (A.15), $B^{\prime}-B \in \boldsymbol{S}_{1}$. Therefore the following expression is well defined on $\rho(\widetilde{A}) \cap \rho\left(A_{0}\right)$

$$
\Delta_{\widetilde{A}}^{\Pi} / \widetilde{A}(z)=\operatorname{det}\left(I+\left(B^{\prime}-B\right)(B-M(z))^{-1}, \quad z \in \rho(\widetilde{A}) \cap \rho\left(A_{0}\right) .\right.
$$


It is called the perturbation determinant of the pair $\left\{\widetilde{A}^{\prime}, \widetilde{A}\right\}$ with respect to $\Pi$, see [MN1]. The following formula plays the key role in what follows

$$
\left(\Delta_{\widetilde{A}^{\prime}}^{\Pi} / \widetilde{A}(z)\right)^{-1} \frac{d}{d z} \Delta_{\widetilde{A}^{\prime} / \widetilde{A}}^{\Pi}(z)=\operatorname{trace}\left(\left(\widetilde{A}^{\prime}-z I\right)^{-1}-(\widetilde{A}-z I)^{-1}\right), \quad z \in \rho(\widetilde{A}) \cap \rho\left(\widetilde{A}^{\prime}\right) .
$$

Let now $\left\{\widetilde{A}^{\prime \prime}, \widetilde{A^{\prime}}, \widetilde{A}\right\}$ be a triple of proper extensions of $A$ such that each pair is resolvent comparable. Then the following three perturbation determinants are well defined and

$$
\Delta_{\widetilde{A}^{\prime \prime} / \widetilde{A}^{\prime}}^{\prod_{(z)}}(z) \Delta_{\widetilde{A}^{\prime} / \widetilde{A}}^{\prod}(z)=\Delta_{\widetilde{A}^{\prime \prime} / \widetilde{A}}^{\prod_{\widetilde{A}}}(z), \quad z \in \rho(\widetilde{A}) \cap \rho\left(\widetilde{A}^{\prime}\right) \cap \rho\left(\widetilde{A}^{\prime \prime}\right) .
$$

A.4 The existence of a spectral shift function. Now we are ready to improve Theorem 3.13 from [MN2].

Theorem A.6. Let $\left\{L_{1}, L_{0}\right\}$ be a pair of m-accumulative resolvent comparable operators. Suppose also that $\left(\rho\left(L_{0}\right) \cup \sigma_{\mathrm{c}}\left(L_{0}\right)\right) \cap \mathbb{C}_{-} \neq \varnothing$. Then

(i) There is a symmetric operator $A, n_{+}(A)=n_{-}(A)$, such that $L_{1}, L_{0} \in \operatorname{Ext}_{A}$. Moreover, there is a boundary triplet $\Pi=\left\{\mathscr{K}, \Gamma_{0}, \Gamma_{1}\right\}$ for $A^{*}$, regular for the pair $\left\{L_{1}, L_{0}\right\}$.

(ii) For each (not necessarily regular) boundary triplet $\Pi$, the perturbation determinant $\Delta_{L_{1} / L_{0}}^{\Pi}$ admits a (non-unique) representation

$$
\Delta_{L_{1} / L_{0}}^{\Pi}(z)=c \exp \left(\int_{\mathbb{R}}\left((t-z)^{-1}-t\left(1+t^{2}\right)^{-1}\right) \boldsymbol{\omega}_{\mathrm{a}}(t) d t\right), \quad z \in \mathbb{C}_{+} .
$$

with a complex function $\boldsymbol{\omega}_{\mathrm{a}} \in L^{1}\left(\mathbb{R},\left(1+t^{2}\right)^{-1}\right)$ and a constant $c \in \mathbb{C}$.

(iii) If $\boldsymbol{\omega}_{a}$ satisfies (A.17), then $\boldsymbol{\omega}_{\mathrm{a}} \in \mathscr{S}\left\{L_{1}, L_{0}\right\}$, i.e., the following formula holds:

$$
\operatorname{trace}\left(\left(L_{1}-z I\right)^{-1}-\left(L_{0}-z I\right)^{-1}\right)=-\int_{\mathbb{R}}(t-z)^{-2} \boldsymbol{\omega}_{\mathrm{a}}(t) d t, \quad z \in \mathbb{C}_{+} .
$$

(iv) If $L_{0}$ (respectively, $L_{1}$ ) is self-adjoint, then there is $\boldsymbol{\omega}_{\mathrm{a}} \in \mathscr{S}\left\{L_{1}, L_{0}\right\}$ satisfying $\operatorname{Im}\left(\boldsymbol{\omega}_{\mathrm{a}}(t)\right) \leq 0$ (respectively, $\operatorname{Im}\left(\boldsymbol{\omega}_{\mathrm{a}}(t)\right) \geq 0$ ) a.e. on $\mathbb{R}$.

(v) If $\boldsymbol{\xi}_{\mathrm{a}} \in \mathscr{S}\left\{L_{1}, L_{0}\right\}$ is real-valued, then for almost all $x \in \mathbb{R}$,

$$
\boldsymbol{\xi}_{\mathrm{a}}(x)=\frac{1}{\pi} \lim _{y \downarrow 0} \operatorname{Im}\left(\log \left(\Delta_{L_{1} / L_{0}}^{\Pi}(x+i y)\right)\right) .
$$

Proof. (i). We put

$$
A f=L f=L_{0} f, \quad f \in \operatorname{dom}(A)=\operatorname{dom}\left(L_{1}\right) \cap \operatorname{dom}\left(L_{1}^{*}\right) \cap \operatorname{dom}\left(L_{0}\right) \cap \operatorname{dom}\left(L_{0}^{*}\right) .
$$

Clearly, $A$ is a closed symmetric operator, $L_{1}$ and $L_{0}$ are its proper extensions. Moreover, the assumption $\left(\rho\left(L_{0}\right) \cup \sigma_{\mathrm{c}}\left(L_{0}\right)\right) \cap \mathbb{C}_{-} \neq \varnothing$ implies that $n_{+}(A)=n_{-}(A)$. Assume for simplicity that $A$ is densely defined. Since $\left(\rho\left(L_{0}\right) \cup \sigma_{c}\left(L_{0}\right)\right) \cap \mathbb{C}_{-} \neq \varnothing$ and $\rho\left(L_{0}\right) \cap \rho\left(L_{1}\right) \supset$ $\mathbb{C}_{+}$, the extensions $L_{0}$ and $L_{1}$ meet the hypotheses of Theorem A.5. Thus, there exists a boundary triplet $\Pi=\left\{\mathcal{H}, \Gamma_{0}, \Gamma_{1}\right\}$ for $A^{*}$ regular for the pair $\left\{L_{1}, L_{0}\right\}$, i.e. such that

$$
L_{1}=A_{B^{\prime}}=A^{*} \mid \operatorname{Ker}\left(\Gamma_{1}-B^{\prime} \Gamma_{0}\right) \quad \text { and } \quad L_{0}=A_{B}=A^{*} \mid \operatorname{Ker}\left(\Gamma_{1}-B \Gamma_{0}\right)
$$

where the boundary operators $B^{\prime}, B$ are in $\mathcal{B}(\mathscr{K})$ (see (A.12)).

(ii) By Lemma A.4 (ii), $B^{\prime}$ and $B$ are accumulative because so are $A_{B^{\prime}}$ and $A_{B}$. Besides, $B^{\prime}-B \in \boldsymbol{S}_{1}$ because $\left(L_{1}-\mathrm{i} I\right)^{-1}-\left(L_{0}-\mathrm{i} I\right)^{-1} \in \boldsymbol{S}_{1}$. 
We set $B^{\prime \prime} \stackrel{\text { def }}{=} \operatorname{Re} B^{\prime}+\mathrm{i} \operatorname{Im} B$ and $L_{2} \stackrel{\text { def }}{=} A^{*} \mid \operatorname{Ker}\left(\Gamma_{1}-B^{\prime \prime} \Gamma_{0}\right)=A_{B^{\prime \prime}}$. Clearly, $L_{2} \in$ Ext $A$ and by Lemma A.4 (ii), it is $m$-accumulative because so is $B^{\prime \prime}$. Since $B^{\prime}-B^{\prime \prime}=$ $\mathrm{i}\left(\operatorname{Im} B^{\prime}-\operatorname{Im} B\right) \in \boldsymbol{S}_{1}$, the perturbation determinant

$$
\Delta_{L_{1} / L_{2}}^{\Pi}(z)=\operatorname{det}\left(I+\mathrm{i}\left(\operatorname{Im} B^{\prime}-\operatorname{Im} B\right)\left(B^{\prime \prime}-M(z)\right)^{-1}\right), \quad z \in \mathbb{C}_{+},
$$

is well defined. Here $M$ is the Weyl function corresponding to $\Pi$. Since $\operatorname{Im} B^{\prime}-\operatorname{Im} B \leq$ $-\operatorname{Im} B=-\operatorname{Im} B^{\prime \prime}$, Lemma A.2 (ii) guarantees a representation

$$
\Delta_{L_{1} / L_{2}}^{\Pi}(z)=\varkappa \exp \left(\int_{\mathbb{R}}\left((t-z)^{-1}-t\left(1+t^{2}\right)^{-1}\right) \eta(t) d t\right), \quad z \in \mathbb{C}_{+},
$$

for a real-valued function $\eta$ in $L^{2}\left(\mathbb{R},\left(1+t^{2}\right)^{-1}\right)$ and $\varkappa \in \mathbb{T}$.

Next, since $B^{\prime \prime}-B=\operatorname{Re} B^{\prime}-\operatorname{Re} B \in \boldsymbol{S}_{1}$, the determinant $\Delta_{L_{2} / L_{0}}^{\Pi}$ is well defined. Since $\operatorname{Re} B^{\prime}-\operatorname{Re} B=\left(\operatorname{Re} B^{\prime}-\operatorname{Re} B\right)^{*}$, by Lemma A.3 (ii), we obtain a representation

$$
\begin{aligned}
& \Delta_{L_{2} / L_{0}}^{\Pi}(z)=\operatorname{det}\left(I+\left(\operatorname{Re} B^{\prime}-\operatorname{Re} B\right)(B-M(z))^{-1}\right) \\
& \quad=k \exp \left(\int_{\mathbb{R}}\left((t-z)^{-1}-t\left(1+t^{2}\right)^{-1}\right) \xi(t) d t\right), \quad z \in \mathbb{C}_{+},
\end{aligned}
$$

with a real function $\xi$ in $L^{2}\left(\mathbb{R},\left(1+t^{2}\right)^{-1} d t\right)$ and $k>0$. Combining (A.21) with (A.22), using the chain rule, and setting $\boldsymbol{\omega}_{\mathrm{a}} \stackrel{\text { def }}{=} \xi+i \eta$, we arrive at (A.17) with $c=k \varkappa$.

(iii) Formula (A.18) is an immediate consequence of (A.17) and (A.16).

(iv) Let $L_{0}=L_{0}^{*}$. Then $B=B^{*}$ and $B^{\prime \prime}=\operatorname{Re} B^{\prime}=\left(B^{\prime \prime}\right)^{*}$. Hence $L_{2}=A_{B^{\prime \prime}}=L_{2}^{*}$ and $\operatorname{Im} B^{\prime}-\operatorname{Im} B=\operatorname{Im} B^{\prime} \leq \mathbf{0}$. By Lemma A.2 (i) with $V_{+}=\left|\operatorname{Im} B^{\prime}\right|$ and $B=B^{\prime}$, we have $\Delta_{L_{1} / L_{2}}^{\Pi}(z)=\left(\Delta_{L_{2} / L_{1}}^{\Pi}(z)\right)^{-1}=\bar{\varkappa} \exp \left(-\int_{\mathbb{R}}\left((t-z)^{-1}-t\left(1+t^{2}\right)^{-1}\right) \eta_{+}(t) d t\right), \quad z \in \mathbb{C}_{+}$, where $\eta_{+}(t) \geq 0$ a.e. on $\mathbb{R}$. Combining this identity with (A.22), applying the chain rule for determinants, and setting $\boldsymbol{\omega}_{\mathrm{a}}(t) \stackrel{\text { def }}{=} \xi(t)-\mathrm{i} \eta_{+}(t)$, we arrive at (A.17) with $\operatorname{Im}\left(\boldsymbol{\omega}_{\mathrm{a}}(t)\right) \leq$ $0, t \in \mathbb{R}$, and $\varkappa \in \mathbb{T}$. The case $L_{1}=L_{1}^{*}$ is similar.

(v) Substituting $\boldsymbol{\xi}_{\mathrm{a}}$ in (A.17) in place of $\boldsymbol{\omega}_{\mathrm{a}}$ and taking the imaginary part of the logarithms of both sides, we obtain

$$
\operatorname{Im}\left(\log \left(\Delta_{L_{1} / L_{0}}^{\Pi}(x+\mathrm{i} y)\right)\right)=\int_{\mathbb{R}} y\left((t-x)^{2}+y^{2}\right)^{-1} \boldsymbol{\xi}_{\mathrm{a}}(t) d t+\operatorname{Im} c .
$$

Applying the Fatou theorem as $y \downarrow 0$ we arrive at (A.19).

Remark. 1. Note that definition (A.16) is a special case of a more general definition introduced in [MN1] which allows us to treat the case of pairs $\left\{A_{B}, A_{B^{\prime}}\right\}\left(\subset \operatorname{Ext}_{A}\right)$ with unbounded closed boundary operators $B, B^{\prime}$ satisfying the following conditions:

(i) $\operatorname{dom} B=\operatorname{dom} B^{\prime}$;

(ii) $\left(B^{\prime}-B\right)(B-M(z))^{-1} \in \boldsymbol{S}_{1} \quad$ for $\quad z \in \rho\left(A_{B}\right) \cap \rho\left(A_{0}\right)$.

In this case the operators $A_{B}$ and $A_{B^{\prime}}$ are resolvent comparable.

This definition allows us to extend Lemmata A.2 and A.3 to the case of unbounded maccumulative operators $B$ (in Lemma A.2 it is required in addition to this that $\operatorname{dom} B=$ $\left.\operatorname{dom} B^{*}\right)$. 
Note also that the choice of $A=\mathbf{0}$ in (A.20) allows us to treat the case of trace class additive perturbations in the framework of the above definition. Indeed, in this case a boundary triplet $\Pi=\left\{\mathscr{K}, \Gamma_{0}, \Gamma_{1}\right\}$ can be chosen so that $M(z)=z, A_{B}=B$, and $A_{B^{\prime}}=B^{\prime}$, and condition (i) implies that $A_{B^{\prime}}=A_{B}+V$, where $V=\cos \left(B^{\prime}-B\right)$. Now definition (A.16) coincides with the classical definition

$$
\Delta_{B^{\prime} / B}(z)=\operatorname{det}\left(I+\left(B^{\prime}-B\right)(B-z)^{-1}\right), \quad z \in \rho(B),
$$

of the perturbation determinant for the pair $\left\{B^{\prime}, B\right\}, B^{\prime}=B+V$, (see [Kr1]-[Kr4], [Ya]).

2. In this case $\left(A=\mathbf{0}, M(z)=z, A_{B}=B, A_{B^{\prime}}=B^{\prime}\right)$ formula (A.8) takes the form

$\operatorname{det}\left(I+\left(B^{\prime}-B\right)(B-z)^{-1}\right)=c \exp \left(\frac{1}{\pi} \int_{\mathbb{R}}\left(\frac{1}{t-z}-\frac{t}{1+t^{2}}\right) \boldsymbol{\xi}(t) d t\right), \quad z \in \mathbb{C}_{+}$.

If in addition to this $V=V^{*} \in \boldsymbol{S}_{1}$, it can be shown (see the proof of Lemma 5.3 of [MN2]) that $\boldsymbol{\xi} \in L^{1}(\mathbb{R})$ and formula (A.23) is reduced to $\Delta_{B^{\prime} / B}(z)=\exp \left(\frac{1}{\pi} \int_{\mathbb{R}}(t-z)^{-1} \boldsymbol{\xi}(t) d t\right)$. So, if $B=B^{*}$, Lemma A.3 coincides with the Krein classical result proved originally in [Kr1] by using the step method (see also [Ya]).

3. Note that in the case of additive trace class perturbations the proof of Theorem A.6 is simplified and the technique of boundary triplets is unnecessary.

Note in conclusion that several papers are devoted to trace formulae for pairs $\{-\Delta, \Delta+$ $q$, where $\Delta$ is the Laplacian operator either in $L^{2}\left(\mathbb{R}^{n}\right)$, or on a discrete lattice and $q$ is a complex-valued potential (see e.g. [KL] and references therein).

\section{REFERENCES}

[AN] V.M. AdAmJAn and H. NEIDHARDt, On the summability of the spectral shift function for pair of contractions and dissipative operators, J. Operator Th. 24 (1990), 187-205.

[AP1] A.B. Aleksandrov and V.V. Peller, Operator Lipschitz functions, Russian Mathematical Surveys 71:4 (2016), 605-702.

[AP2] A.B. Aleksandrov and V.V. Peller, Krein's trace formula for unitary operators and operator Lipschitz functions, Funct. Anal and Appl., 50:3 (2016), 167-175.

[BS1] M.S. Birman and M.Z. Solomyak, Double Stieltjes operator integrals, Problems of Math. Phys., Leningrad. Univ. 1 (1966), 33-67 (Russian). English transl.: Topics Math. Physics 1 (1967), 25-54, Consultants Bureau Plenum Publishing Corporation, New York.

[BS2] M.S. Birman and M.Z. Solomyak, Remarks on the spectral shift function, Zapiski Nauchn. Semin. LOMI 27 (1972), 33-46 (Russian). English transl.: J. Soviet Math. 3 (1975), 408-419.

[DK] YU.L. DALETSKII and S.G. KREIN, Integration and differentiation of functions of Hermitian operators and application to the theory of perturbations (Russian), Trudy Sem. Functsion. Anal., Voronezh. Gos. Univ. 1 (1956), 81-105.

[DM] V.A. DeRKACh AND M.M. Malamud, Generalized resolvents and the boundary value problems for Hermitian operators with gaps, J. Funct. Anal., 95 (1991), 1-95.

[F] Yu.B. FARForovskayA, An example of a Lipschitzian function of selfadjoint operators that yields a nonnuclear increase under a nuclear perturbation. Zap. Nauchn. Sem. Leningrad. Otdel. Mat. Inst. Steklov. (LOMI) 30 (1972), 146-153 (Russian).

[GMN] F. Gesztezy, K.A. Makarov, and S.N. NAвоко, The spectral shift operator, In: Mathematical results in quantum mechanics (Prague, 1998), Oper. Theory Adv. Appl., 108, 59-90. Birkhäuser, Basel, 1999.

[JW] B.E. Johnson and J.P. Williams, The range of a normal derivation, Pacific J. Math. 58 (1975), 105-122. 
[KS] E. Kissin and V. S. Shulman, Classes of operator-smooth functions. I Operator-lipschitz functions, Proc. Edinburgh Math. Soc. 48 (2005), 151-173.

[Ko] P. Koosis, Introduction to $H_{p}$ spaces, London Mathematical Society Lecture Note Series, v. 40, Cambridge University Press, Cambridge, 1980.

[KL] E. Korotyaev and A. LAPTEV, Trace formulae for Schrodinger operators with complex-valued potentials on cubic lattices, arXiv:1609.09703v1 [math.SP], 30 Sep 2016.

[Kr1] M.G. KREIN, On a trace formula in perturbation theory, Mat. Sbornik 33 (1953), 597-626 (Russian).

[Kr2] M.G. KReIn, On perturbation determinants and a trace formula for unitary and self-adjoint operators, Dokl. Akad. Nauk SSSR 144:2 (1962), 268-271(Russian).

[Kr3] M. G. Krel̆. Some new studies in the theory of perturbations of self-adjoint operators. In First Math. Summer School, Part I (Russian), pages 103-187. Izdat. "Naukova Dumka", Kiev, 1964.

[Kr4] M.G. KReIn, Perturbation determinants and a trace formula for some classes of pairs of operators, J. Operator Th., 17 (1987), 129-187.

[L] I.M. Lifshitz, On a problem in perturbation theory connected with quantum statistics, Uspekhi Mat. Nauk 7 (1952), 171-180 (Russian).

[MN1] M. Malamud and H. Neidhardt, Perturbation determinants for singular perturbations, Russian J. of Math. Phys., 21:1 (2014), 55-98.

[MN2] M. Malamud and H. NeIDhardt, Trace formulas for additive and non-additive perturbations, Adv. Math. 274 (2015), 736-832, Preprint, arXiv:1212.6887 [math-ph], 2012.

[MSZ] K. Makarov, A. Skripka and M. Zinchenko, On a perturbation determinant for accumulative operators, Integral Equations Operator Theory 81 (2015), 301317.

[Pe1] V.V. PELleR, Hankel operators in the theory of perturbations of unitary and self-adjoint operators, Funktsional. Anal. i Prilozhen. 19:2 (1985), 37-51 (Russian). English transl.: Funct. Anal. Appl. 19 (1985), 111-123.

[Pe2] V.V. Peller, For which $f$ does $A-B \in \mathbf{S}_{p}$ imply that $f(A)-f(B) \in \mathbf{S}_{p}$ ?, Operator Theory, Birkhäuser, 24 (1987), 289-294.

[Pe3] V.V. PELler, Hankel operators in the perturbation theory of of unbounded self-adjoint operators. Analysis and partial differential equations, 529-544, Lecture Notes in Pure and Appl. Math., 122, Dekker, New York, 1990.

[Pe4] V.V. PELler, Differentiability of functions of contractions, In: Linear and complex analysis, AMS Translations, Ser. 2226 (2009), 109-131, AMS, Providence.

[Pe5] V.V. Peller, The Lifshits-Krein trace formula and operator Lipschitz functions, Proc. Amer. Math. Soc. 144 (2016), 5207-5215.

[R1] A. V. RyBKin, The spectral shift function for a dissipative and a selfadjoint operator, and trace formulas for resonances, Mat. Sb. (N.S.), 125(167):3 (1984), 420-430.

[R2] A.V. RyBkin, A trace formula for a contractive and a unitary operator, Funktsional. Anal. i Prilozhen., 21:4 (1987), 85-87.

[R3] A.V. RYBKIn, The discrete and the singular spectrum in the trace formula for a contractive and a unitary operator, Funktsional. Anal. i Prilozhen., 23:3 (1989), 84-85.

[R4] A.V. RYBKIN, The spectral shift function, the characteristic function of a contraction and a generalized integral, Mat. Sb. 185(10) (1994), 91-144.

[SNF] B. Sz.-NAGY AND C. FoiAs, Harmonic analysis of operators on Hilbert space, Akadémiai Kiadó, Budapest, 1970.

[Ya] D. R. Yafaev. Mathematical scattering theory, volume 105 of Translations of Mathematical Monographs. American Mathematical Society, Providence, RI, 1992.

[Yo] K. YosidA, Functional Analysis, Springer-Verlag, Berlin, 1980. 
M.M. Malamud

Institute of Applied

Mathematics and Mechanics

NAS of Ukraine

Slavyansk

Ukraine
H. Neidhardt

Institut für Angewandte

Analysis und Stochastik

Mohrenstr. 39

D-10117 Berlin

Germany
V.V. Peller

Department of Mathematics

Michigan State University

East Lansing, Michigan 48824

USA 